\title{
Extremely imbalanced two-dimensional electron-hole-photon systems
}

\author{
A. Tiene $\odot,,^{1, *}$ J. Levinsen $\odot,{ }^{2,3}$ M. M. Parish $\odot,{ }^{2,3}$ A. H. MacDonald, ${ }^{4}$ J. Keeling $\odot,{ }^{5}$ and F. M. Marchetti $\odot^{1, \dagger}$ \\ ${ }^{1}$ Departamento de Física Teórica de la Materia Condensada \& Condensed Matter Physics Center (IFIMAC), \\ Universidad Autónoma de Madrid, Madrid 28049, Spain \\ ${ }^{2}$ School of Physics and Astronomy, Monash University, Victoria 3800, Australia \\ ${ }^{3}$ ARC Centre of Excellence in Future Low-Energy Electronics Technologies, Monash University, Victoria 3800, Australia \\ ${ }^{4}$ Department of Physics, The University of Texas at Austin, Austin, Texas 78712, USA \\ ${ }^{5}$ SUPA, School of Physics and Astronomy, University of St Andrews, St Andrews KY16 9SS, United Kingdom
}

(Received 21 November 2019; accepted 11 March 2020; published 28 April 2020)

\begin{abstract}
We investigate the phases of two-dimensional electron-hole systems strongly coupled to a microcavity photon field in the limit of extreme charge imbalance. Using variational wave functions, we examine the competition between different electron-hole paired states for the specific cases of semiconducting III-V single quantum wells, electron-hole bilayers, and transition-metal dichalcogenide monolayers embedded in a planar microcavity. We show how the Fermi sea of excess charges modifies both the electron-hole bound state (exciton) properties and the dielectric constant of the cavity active medium, which in turn affects the photon component of the many-body polariton ground state. On the one hand, long-range Coulomb interactions and Pauli blocking of the Fermi sea promote electron-hole pairing with finite center-of-mass momentum, corresponding to an excitonic roton minimum. On the other hand, the strong coupling to the ultra-low-mass cavity photon mode favors zeromomentum pairs. We discuss the prospect of observing different types of electron-hole pairing in the photon spectrum.
\end{abstract}

DOI: 10.1103/PhysRevResearch.2.023089

\section{INTRODUCTION}

Recent technological progress has opened up the possibility to study the interplay between strong light-matter coupling and electronic doping in semiconductor structures [1-8]. Here, there is the prospect of generating and controlling novel strongly correlated phases involving photons, electron-hole pairs, and an electron gas. Electron-hole systems with charge imbalance are expected to display exotic pairing phenomena such as the spontaneous appearance of electron-hole pairs (excitons) with finite center-of-mass (COM) momentum [9-12]. This finite COM paired state is equivalent to the FuldeFerrell-Larkin-Ovchinnikov (FFLO) phase [13,14], a spatially modulated paired phase first proposed in the context of spinimbalanced conventional superconductors. The study of this inhomogeneous superfluid phase has attracted noticeable interest over the past five decades in a wide range of physical systems - see, e.g., the recent reviews in Refs. [15-17]. However, a conclusive experimental observation of the FFLO state remains a challenge. Signatures and indirect evidence of the FFLO phase have been reported in heavy-fermion systems [18], layered organic superconductors [19-23], and

\footnotetext{
*antonio.tiene@uam.es

†francesca.marchetti@uam.es

Published by the American Physical Society under the terms of the Creative Commons Attribution 4.0 International license. Further distribution of this work must maintain attribution to the author(s) and the published article's title, journal citation, and DOI.
}

iron-based superconductors [24]. There has also been related work on ultracold gases in one-dimensional (1D) optical lattices, paving the way toward studying FFLO states in such systems [25]. It is therefore of particular interest to understand how such a state in an electron-hole system might be probed and controlled with light.

One particularly interesting class of materials giving access to this regime are transition-metal dichalcogenide (TMDC) monolayers [26]. These structures are characterized by distinctive excitonic effects, ascribed to two-dimensional (2D) confinement and weak dielectric screening of the carrier Coulomb interactions in the 2D limit $[27,28]$. Coupling between excitons and electrically injected free charge carriers has been recently demonstrated (see, e.g., Ref. [29]), together with the realization of electron-hole bilayers with independently tunable carrier densities [30]. Further, the large exciton binding energies and strong light-matter coupling of these materials grant the possibility of accessing polaritonic (exciton-photon superposition) phenomena at room temperature. Indeed, the strong light-matter coupling regime has been recently achieved by embedding a TMDC monolayer into an optical microcavity [31], enabling the observation of valleypolarized exciton polaritons at room temperature [32-34]. Such structures provide an ideal environment in which to investigate the interplay between strong light-matter coupling and electronic doping because of the possibility of externally tuning the electron density and light-matter coupling [7].

Imbalanced electron-hole-photon systems may also be realized using III-V and II-VI semiconducting single or coupled quantum wells. In particular, double quantum wells with independent electrical contacts, which allow one to 
independently tune the electron and hole densities in each layer, have been realized [35-37]. Here, the 2D electron and hole gases are separated by a barrier that is high enough to prevent recombination while thin enough to allow interlayer exciton formation. Such gated structures have not yet been embedded in a microcavity, so they have not yet been studied in the strong light-matter coupling regime. However, a 2D electron gas (2DEG) in a single quantum well embedded into a planar microcavity has been realized experimentally. Indeed, such a device can be produced either optically, as shown for GaAs-based quantum-well structures in Refs. [2-4], or by using a modulation-doped CdTe [1] and GaAs [5,6] quantum well embedded in a planar cavity. In these structures, at low 2DEG density, the negatively charged exciton-polariton, corresponding to a superposition of a trion (two electrons and one hole) and a cavity photon, emerges as a dominant feature in the system spectrum. At larger densities, this physics is expected to evolve into that of the Fermi-edge exciton polariton, as described in Refs. [38,39] and references therein. A connected problem is that of the Fermi-polaron polaritons [40]. Recent spectroscopic measurements in a gate-tunable monolayer $\mathrm{MoSe}_{2}$ embedded into an open microcavity structure [7] have shown strong signatures of both trion and polaron resonances, where a mobile impurity, e.g., an optically generated hole, is dressed by particle-hole excitations across the 2DEG Fermi surface.

In this paper, we discuss pairing effects in strongly carrier density imbalanced electron-hole 2D structures strongly coupled to a microcavity photon field. In the absence of light, it was previously shown that a sufficiently high density of excess charge causes the exciton energy to develop a roton minimum at finite CoM momentum [10,12] that is related to the FFLO $[13,14]$ phase first proposed for conventional superconductors. Here, we study how strong coupling to light affects this excitonic FFLO roton minimum. While long-range Coulomb interactions and Pauli blocking promote the formation of a finite CoM momentum bound state, the strong coupling to low mass cavity photons tends to suppress such a phase. Conversely, the formation of an FFLO phase suppresses the coupling to light. We study the competition between these processes by deriving the phase diagram of the equilibrium extremely imbalanced electron-hole-photon system, focusing solely on pairing phenomena. We show that the exciton mode is affected not only by the presence of the majority species Fermi sea, but, at the same time, the excess charge modifies the dielectric constant of the active medium, and thus it also affects the energy of the cavity photon mode. Consequences of this predicted energy shift of the photon mode in the presence of a Fermi sea can be observed by comparing structures with different light-matter coupling, e.g., by embedding a different number of quantum wells into the planar cavity and thus in effect changing the Rabi splitting.

The paper is organized as follows: In Sec. II we introduce the electron-hole-photon system that we consider, its Hamiltonian, and the renormalization of the cavity photon energy in the presence of an active medium (Sec. II A), i.e., a single or double quantum well embedded into a planar microcavity. In Sec. III, we describe the variational approach we employ to describe the extremely imbalanced electron-hole-photon system. The paired (bound) and normal (unbound) polariton phases we consider are described in Secs. III A and III B, respectively. Results for the case of III-V structures are described in Sec. IV, while the specific case of doped TMDC monolayers embedded into a planar cavity is discussed in Sec. V. Conclusions and perspectives are gathered in Sec. VI. Additional information related to this work can be found in the Appendixes.

\section{MODEL}

We consider an electron-hole system in either a bilayer or a single-layer geometry, embedded in a planar cavity. We consider the spin-polarized case, where electrons and holes are in a single spin state (e.g., by introducing external magnetic field). The system can be described by the following Hamiltonian (in the following, we set $\hbar=1$ ):

$$
\begin{aligned}
& \hat{H}=\hat{H}_{0}+\hat{H}_{\text {Coul }}+\hat{H}_{\text {e-h-C }} \text {, } \\
& \hat{H}_{0}=\sum_{\mathbf{k} \sigma}\left(\epsilon_{\mathbf{k}, \sigma}+\frac{E_{g}}{2}\right) \hat{c}_{\mathbf{k}, \sigma}^{\dagger} \hat{c}_{\mathbf{k}, \sigma}+\sum_{\mathbf{q}} v_{\mathrm{Cq}} \hat{a}_{\mathbf{q}}^{\dagger} \hat{a}_{\mathbf{q}}, \\
& \hat{H}_{\text {Coul }}=\sum_{\mathbf{k} \mathbf{k}^{\prime} \mathbf{q}, \sigma \sigma^{\prime}} \frac{W_{\mathbf{q}}^{\sigma \sigma^{\prime}}}{2 \mathcal{A}} \hat{c}_{\mathbf{k}, \sigma}^{\dagger} \hat{c}_{\mathbf{k}^{\prime}, \sigma^{\prime}}^{\dagger} \hat{c}_{\mathbf{k}^{\prime}+\mathbf{q}, \sigma^{\prime}} \hat{c}_{\mathbf{k}-\mathbf{q}, \sigma}, \\
& \hat{H}_{\mathrm{e}-\mathrm{h}-\mathrm{C}}=\frac{g}{\sqrt{\mathcal{A}}} \sum_{\mathbf{k q}}\left(\hat{c}_{\frac{\mathbf{q}}{2}+\mathbf{k}, 1}^{\dagger} \hat{c}_{\frac{\mathbf{q}}{2}-\mathbf{k}, 2}^{\dagger} \hat{a}_{\mathbf{q}}+\text { H.c. }\right) .
\end{aligned}
$$

Here, $\mathcal{A}$ is the system area, which also determines the spacing of allowed momenta $\mathbf{k}, \mathbf{q}$. The operators appearing in this Hamiltonian correspond to cavity photons, $\hat{a}_{\mathbf{q}}$, and fermionic excitations (either electrons or holes, as discussed below), $\hat{c}_{\mathbf{k}, \sigma=1,2}$. The index $\sigma=1$ labels the majority species, and this species has density $n_{1}$ and Fermi energy

$$
E_{\mathrm{F}}=\frac{k_{\mathrm{F}}^{2}}{2 m_{1}}=\frac{2 \pi}{m_{1}} n_{1},
$$

where $k_{F}$ is the Fermi momentum. The index $\sigma=2$ indicates the minority species, for which we have at most one particle. Majority and minority particles correspond to distinct bands-conduction-band electrons and valence-band holes. In addition, for the bilayer geometry [Fig. 1(a)], the two species exist in two distinct quantum wells with transverse separation $d$. For the single-layer geometry [Fig. 1(b)], both species live in the same quantum well. The electrons and holes have dispersions $\epsilon_{\mathbf{k}, \sigma}=\mathbf{k}^{2} / 2 m_{\sigma}$, where $m_{\sigma}$ is the mass, $\mathbf{k}$ is the two-dimensional (2D) momentum, and $E_{g}$ is the band gap. For GaAs, the particle mass ratio is $m_{2} / m_{1}=4$ in the case of a minority hole in a majority Fermi sea of electrons, or $m_{2} / m_{1}=0.25$ for an electron in a Fermi sea of holes.

The bare intra- and interspecies Coulomb interactions for a bilayer geometry involving two inorganic quantum wells, such as III-V structures, are given, respectively, by

$$
\begin{aligned}
& W_{\mathbf{q}}^{\sigma \sigma}=U_{\mathbf{q}} \equiv \frac{2 \pi e^{2}}{\varepsilon q}, \\
& W_{\mathbf{q}}^{12}=W_{\mathbf{q}}^{21}=-V_{\mathbf{q}} \equiv-U_{\mathbf{q}} e^{-q d},
\end{aligned}
$$

where we use Gaussian units $4 \pi \varepsilon_{0}=1$. In the absence of both doping and coupling to light, the Coulomb attraction between one electron and one hole leads to the Schrödinger equation 

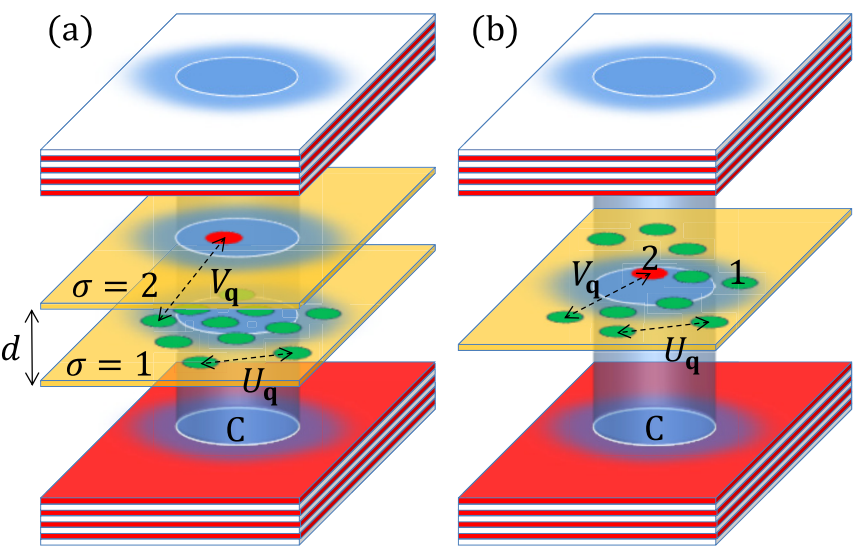

FIG. 1. Schematic representation of the system. (a) Two quantum wells, labeled by the index $\sigma=1,2$ and separated by a distance $d$, form an electron-hole bilayer in the extremely imbalanced limit. The minority species belongs to the $\sigma=2$ layer, while the majority species at $\sigma=1$ forms an interacting Fermi sea-the mass ratio $m_{2} / m_{1}$ establishes which layer is populated by either electrons or holes. $U_{\mathbf{q}}$ and $V_{\mathbf{q}}$ are, respectively, intra- and interspecies Coulomb interactions. The bilayer is located inside a planar cavity that confines the cavity photon mode $(C)$. The (blue) shaded area represents the finite-size external laser pump spot. (b) Same setup in a single quantum-well geometry. Here, the majority $\sigma=1$ and minority $\sigma=2$ species belong to the same well.

for a $2 \mathrm{D}$ exciton [41]:

$$
\left(E-\epsilon_{\mathbf{k}, 1}-\epsilon_{\mathbf{k}, 2}\right) \varphi_{\mathbf{k}}=-\sum_{\mathbf{k}^{\prime}} \frac{V_{\mathbf{k}-\mathbf{k}^{\prime}}}{\mathcal{A}} \varphi_{\mathbf{k}^{\prime}},
$$

where we measure the energy $E$ from the electron-hole band gap $E_{g}$. Here, $\varphi_{\mathbf{k}}$ is the electron-hole wave function at relative momentum $\mathbf{k}$. The negative energy solutions of this equation describe bound states and yield the exciton energies. Of particular interest is the $1 s$ exciton, with wave function $\Phi_{1 s \mathbf{k}}^{(d)}$ and binding energy $\left|E_{\mathrm{X}}^{(d)}\right|$, where $E=E_{\mathrm{X}}^{(d)}<0$ is the lowest energy eigenvalue of Eq. (4). For bilayers at a given separation $d$, the exciton properties can be found by numerically solving the Schrödinger equation (4). However, in the case of a singlelayer geometry $d=0$, where $V_{\mathbf{q}}=U_{\mathbf{q}}$, the Schrödinger equation can be solved analytically, giving the $1 s$ exciton binding energy (or exciton Rydberg) in two dimensions in terms of the Bohr radius $a_{\mathrm{X}}$ and the reduced mass $\mu=m_{1} m_{2} /\left(m_{1}+m_{2}\right)$ :

$$
R_{\mathrm{X}}=\left|E_{\mathrm{X}}^{(d=0)}\right|=\frac{e^{2}}{\varepsilon a_{\mathrm{X}}}, \quad a_{\mathrm{X}}=\frac{\varepsilon}{2 \mu e^{2}} .
$$

In this case, one recovers the known expression for the exciton wave function,

$$
\Phi_{1 s \mathbf{k}}^{(d=0)}=\frac{\sqrt{8 \pi} a_{\mathrm{X}}}{\left[1+\left(k a_{\mathrm{X}}\right)^{2}\right]^{3 / 2}} .
$$

Electrons and holes couple with a strength $g$ to photons via the term $\hat{H}_{\mathrm{e}-\mathrm{h}-\mathrm{C}}(1 \mathrm{~d})$. The bare cavity photon dispersion is that of a passive cavity in the absence of the active medium (in our case a single or a double quantum well),

$$
v_{\mathrm{Cq}}=v_{\mathrm{C} \mathbf{0}}+\frac{\mathbf{q}^{2}}{2 m_{\mathrm{C}}} \text {. }
$$

We fix the photon mass $m_{\mathrm{C}}$ to an experimentally relevant value [42], $m_{\mathrm{C}} \simeq 10^{-4}\left(m_{1}+m_{2}\right)$. In the presence of an active medium, the cavity photon frequency is shifted by the coupling to matter excitations, as we discuss in the next section.

\section{A. Renormalization of the cavity photon energy}

In the presence of both light and matter degrees of freedom, the contact coupling term of our model, $\hat{H}_{\mathrm{e}-\mathrm{h}-\mathrm{C}}$ in Eq. (1d), implies an ultraviolet logarithmic divergence of the ground-state energy [43]. Since the details of the high-momentum physics, such as the band curvature due to the crystal lattice structure, are not included within our low-energy model, we will treat the ultraviolet physics via the process of renormalization. This allows us to deduce universal properties of our system that are independent of microscopic details.

To see how the ultraviolet divergence emerges, it is instructive to first consider the description of lower and upper polaritons within our model. To this end, we follow Ref. [43] and consider the most general superposition of a cavity photon at normal incidence and an electron-hole pair:

$$
\left|\Psi_{\mathbf{0}}\right\rangle=\left(\sum_{\mathbf{k}} \frac{\varphi_{\mathbf{k} \mathbf{0}}}{\sqrt{\mathcal{A}}} \hat{c}_{\mathbf{k}, 1}^{\dagger} \hat{c}_{-\mathbf{k}, 2}^{\dagger}+\alpha_{\mathbf{0}} \hat{a}_{\mathbf{0}}^{\dagger}\right)|0\rangle .
$$

Here, $|0\rangle$ is the vacuum state (i.e., a filled valence band, so a vacuum for valence-band holes), $\alpha_{\mathbf{0}}$ is the photon amplitude, and $\varphi_{\mathbf{k} 0}$ is the electron-hole wave function at relative momentum $\mathbf{k}$ and zero COM momentum. Minimizing $\left\langle\Psi_{\mathbf{0}}\right|(\hat{H}-E-$ $\left.E_{g}\right)\left|\Psi_{\mathbf{0}}\right\rangle$ with respect to the complex amplitudes $\varphi_{\mathbf{k} 0}$ and $\alpha_{\mathbf{0}}$, we obtain the coupled eigenvalue equations for the energy $E$ (measured with respect to the band gap $E_{g}$ ) of the polariton state:

$$
\begin{aligned}
\left(E-\epsilon_{\mathbf{k}, 1}-\epsilon_{\mathbf{k}, 2}\right) \varphi_{\mathbf{k} \mathbf{0}} & =-\sum_{\mathbf{k}^{\prime}} \frac{V_{\mathbf{k}-\mathbf{k}^{\prime}}}{\mathcal{A}} \varphi_{\mathbf{k}^{\prime} \mathbf{0}}+g \alpha_{\mathbf{0}}, \\
\left(E-v_{\mathrm{C} \mathbf{0}}+E_{g}\right) \alpha_{\mathbf{0}} & =\frac{g}{\mathcal{A}} \sum_{\mathbf{k}} \varphi_{\mathbf{k} \mathbf{0}} .
\end{aligned}
$$

Inserting Eq. (9a) in Eq. (9b) and rearranging, we obtain

$$
\begin{aligned}
& \left(E-v_{\mathrm{C} \mathbf{0}}+E_{g}+\frac{g^{2}}{\mathcal{A}} \sum_{\mathbf{k}} \frac{1}{-E+\epsilon_{\mathbf{k}, 1}+\epsilon_{\mathbf{k}, 2}}\right) \alpha_{\mathbf{0}} \\
& =\frac{g}{\mathcal{A}^{2}} \sum_{\mathbf{k}, \mathbf{k}^{\prime}} \frac{V_{\mathbf{k}-\mathbf{k}^{\prime}} \varphi_{\mathbf{k}^{\prime} \mathbf{0}}}{-E+\epsilon_{\mathbf{k}, 1}+\epsilon_{\mathbf{k}, 2}} .
\end{aligned}
$$

The sum on the left-hand side of this equation diverges. If we introduce an ultraviolet momentum cutoff $\Lambda$, the divergence is logarithmic in $\Lambda$. In contrast, the right-hand side is finite when $\Lambda \rightarrow \infty$ [43]. One can easily check this in the exciton limit-i.e., where $g$ is small—using the explicit form of the exciton wave function in a single quantum well, Eq. (6). As a consequence, the photon amplitude $\alpha_{0}$ must approach zero as $1 / \ln \Lambda$ for energies $E+E_{g} \sim v_{\mathrm{C} 0}$, which is a signature that the photon frequency shifts in the presence of an active medium. To have finite answers, it is therefore necessary that $v_{\mathrm{C} 0}$ also diverges as $\ln \Lambda$-i.e., we should write quantities in terms of the renormalized (finite and measurable) photon 
energy [43] $\omega_{\mathrm{C} 0}$ as follows:

$$
\omega_{\mathrm{C} \mathbf{0}}=v_{\mathrm{C} \mathbf{0}}-\frac{g^{2}}{\mathcal{A}} \sum_{\mathbf{k}} \frac{1}{-E_{\mathrm{X}}^{(d)}+\epsilon_{\mathbf{k}, 1}+\epsilon_{\mathbf{k}, 2}},
$$

correct to logarithmic accuracy. Here, we have taken the $1 \mathrm{~s}$ exciton binding energy to be the relevant energy scale $E \simeq$ $E_{\mathrm{X}}^{(d)}$, since we are considering the scenario where the photon is resonantly coupled to the $1 s$ exciton. One can thus define the renormalized photon-exciton detuning at zero momentum,

$$
\delta=\omega_{\mathrm{C} \mathbf{0}}-\left(E_{\mathrm{X}}^{(d)}+E_{g}\right),
$$

where $E_{\mathrm{X}}^{(d)}+E_{g}$ is the actual exciton energy that would be measured spectroscopically. Hence, the logarithmic divergence of Eq. (11) exactly cancels the divergence appearing in Eq. (10) such that, when this is expressed in terms of the dressed photon energy $\omega_{\mathrm{C} 0}$ rather than the bare photon energy $\nu_{\mathrm{C} 0}$, one obtains convergent cutoff independent results.

While the photon frequency is renormalized in the presence of an active medium, the Rabi splitting between the lower and upper polaritons remains finite [43]. In the limit $g \ll a_{X} R_{\mathrm{X}}$, this splitting can be written as

$$
\Omega=\frac{2 g}{\mathcal{A}} \sum_{\mathbf{k}} \Phi_{1 s \mathbf{k}}^{(d)} .
$$

Here, $\Phi_{1 s \mathbf{k}}^{(d)}$ is the ground-state wave function of Eq. (4) at layer separation $d$. Indeed, we see from Eq. (10) that, had we chosen to cancel the logarithmic divergence by taking $g \sim 1 / \sqrt{\ln \Lambda}$, then the right-hand side of that equation would go to zero as $\Lambda \rightarrow \infty$, and we would have had no coupling between light and matter.

One can show [43] that the implementation of the renormalization scheme in Eqs. (9a) and (9b) recovers the coupled exciton-photon oscillator model in the limit $g \ll a_{\mathrm{X}} R_{\mathrm{X}}$. The generalization to finite momentum is straightforward [43], and one finds that the lowest eigenvalue $E+E_{g}$ of Eqs. (9a) and (9b) well matches the one-particle lower polariton (LP) energy expression coming from the coupled oscillator model,

$$
\begin{aligned}
\omega_{\mathrm{LPQ}}= & \frac{\omega_{\mathrm{CQ}}+\left(E_{\mathrm{XQ}}^{(d)}+E_{g}\right)}{2} \\
& -\frac{1}{2} \sqrt{\left[\omega_{\mathrm{CQ}}-\left(E_{\mathrm{XQ}}^{(d)}+E_{g}\right)\right]^{2}+\Omega^{2}},
\end{aligned}
$$

where the exciton is assumed to be a structureless particle. Here, $E_{\mathrm{XQ}}^{(d)}=E_{\mathrm{X}}^{(d)}+\mathbf{Q}^{2} / 2\left(m_{1}+m_{2}\right)$ and $\omega_{\mathrm{CQ}}=\omega_{\mathrm{C} \mathbf{0}}+$ $\mathbf{Q}^{2} / 2 m_{\mathrm{C}}$. As shown in Ref. [43], the definitions of the effective detuning, Eq. (11), and Rabi splitting, Eq. (13), represent a first-order approximation in the expansion parameter $g \ll$ $a_{\mathrm{X}} R_{\mathrm{X}}$ to the experimentally measured detuning and Rabi splitting. An effort to obtain a better estimate of both parameters and a comparison with the approximation carried out here is discussed in Appendix A. There, we employ a definition of detuning and Rabi splitting that is similar to a possible experimental procedure. In particular, we obtain their values by least-squares fitting to match the LP dispersion obtained from the coupled oscillator model, Eq. (14). In this way, we find that the differences between the fitted parameters and those defined in Eqs. (12) and (13) are small. This implies only small quantitative changes in our results below when we push our results beyond the $g \ll R_{\mathrm{X}} a_{\mathrm{X}}$ validity regime of Eqs. (12) and (13).

As in Ref. [43], the renormalization procedure we consider is defined for the case at zero gating/doping $\left(E_{\mathrm{F}}=0\right)$. We then increase the density of majority particles while keeping the other parameters fixed. Interestingly, a TMDC monolayer flake embedded in a planar cavity offers the possibility to measure independently the renormalized photon energy $\omega_{\mathrm{C} 0}$ and compare it to the bare value $v_{\mathrm{C} 0}$. In this structure, the TMDC flake has a reduced size compared to the planar cavity, and thus there are regions where the cavity mode is passive and does not couple to the active medium [31]. Such a measurement would reveal that in the real system, the energy correction due to dressing is actually finite. That is, an effective UV cutoff does indeed exist associated with the nature of electronic states at large momenta; however, this cutoff is a high-energy effect, beyond the scope of our low-energy Hamiltonian.

The definitions we adopt above for renormalization-i.e., how we choose to calibrate the definitions of detuningmatch what we anticipate as a typical experimental protocol. Specifically, it corresponds to a process where, in the absence of gating/doping, i.e., at $E_{\mathrm{F}}=0$, one deduces the photonexciton detuning $\delta$ and the Rabi splitting $\Omega$ by fitting the single-particle polariton dispersion measured in the optical pumping linear regime via a coupled oscillator model. After fixing these experimental conditions, one then increases $E_{\mathrm{F}}$ by doping or gating. The Rabi splitting $\Omega$ can be changed by considering microcavities with different numbers of embedded quantum wells [44]. The detuning $\delta$ can be changed because of the cavity mirror wedge and thus by changing the location of the optical pump spot. Crucially, the value of $\delta$ we use is defined as that measured in the absence of doping or gating before increasing $E_{\mathrm{F}}$-i.e., we assume a definition of $\delta$ that does not vary with doping.

\section{B. Screening}

In writing the Coulomb interaction above, we so far considered the bare Coulomb interaction. However, as we consider a system with electronic doping, these electrons can screen and thus modify the Coulomb interaction. As explained in Sec. III, the screening of Coulomb interactions causes, in the absence of photons, a transition from bound to unbound excitonic states when the majority species density increases [10]. With the aim of including the possibility of describing the binding-unbinding transition, we will present results for both the unscreened case and for screened Coulomb interactions within the static random phase approximation (RPA). In the RPA, the intraspecies potential reads

$$
\begin{aligned}
U_{\mathbf{q}}^{\mathrm{sc}} & =\frac{U_{\mathbf{q}}}{1-U_{\mathbf{q}} \Pi_{1}(\mathbf{q})}, \\
\Pi_{1}(\mathbf{q}) & =\frac{N_{s} m_{1}}{2 \pi}\left[\frac{\sqrt{q^{2}-4 k_{\mathrm{F}}^{2}}}{q} \theta\left(q-2 k_{\mathrm{F}}\right)-1\right],
\end{aligned}
$$

with $N_{s}=1$ for the spin-polarized case. As before, the interspecies potential is then found by $V_{\mathbf{q}}^{\text {sc }}=U_{\mathbf{q}}^{\text {sc }} e^{-q d}$. We expect 
the RPA to provide a good approximation when the exciton Bohr radius greatly exceeds the interparticle spacing of the majority species, i.e., $a_{\mathrm{X}}^{2} n_{1} \gg 1$. In the opposite limit, $a_{\mathrm{X}}^{2} n_{1} \ll$ 1 , screening is negligible. With this in mind, unscreened and RPA screened interactions represent extreme limiting cases, thus allowing us to place a bound on the effect of screening in a realistic material.

\section{VARIATIONAL APPROACH TO THE IMBALANCED ELECTRON-HOLE-PHOTON SYSTEM}

As described in the Introduction, the aim of this paper is to understand how strong light-matter coupling affects the transition from excitons with zero to finite COM momentum, as one varies the majority species density. To address this, we focus on the extreme limit, where there is a single minority particle $\sigma=2$ interacting with a Fermi liquid of majority particles $\sigma=1$ via both Coulomb attraction and the cavity mode. To determine the zero-temperature phase diagram, we find the ground state by a variational approach. The variational state we consider describes a superposition of a photon and an electron-hole pair, on top of a Fermi sea of majority particles, $|\mathrm{FS}\rangle=|\mathrm{FS}\rangle_{1} \otimes|0\rangle_{2} \otimes|0\rangle_{\mathrm{C}}$ :

$$
\left|\Psi_{\mathbf{Q}}\right\rangle=\left(\sum_{\mathbf{k}>k_{\mathrm{F}}} \frac{\varphi_{\mathbf{k} \mathbf{Q}}}{\sqrt{\mathcal{A}}} \hat{c}_{\mathbf{k}, 1}^{\dagger} \hat{c}_{\mathbf{Q}-\mathbf{k}, 2}^{\dagger}+\alpha_{\mathbf{Q}} \hat{a}_{\mathbf{Q}}^{\dagger}\right)|\mathrm{FS}\rangle .
$$

Here, $\varphi_{\mathbf{k} \mathbf{Q}}$ and $\alpha_{\mathbf{Q}}$ are the excitonic and photonic variational parameters, respectively, and the normalization condition requires that $\left\langle\Psi_{\mathbf{Q}} \mid \Psi_{\mathbf{Q}}\right\rangle=\mathcal{A}^{-1} \sum_{\mathbf{k}>k_{\mathrm{F}}}\left|\varphi_{\mathbf{k} \mathbf{Q}}\right|^{2}+\left|\alpha_{\mathbf{Q}}\right|^{2}=1$. The momentum $\mathbf{Q}$ is the COM momentum of the polaritonic bound state, while the label $\mathbf{k}$ denotes the relative electronhole momentum. Pauli blocking forbids occupation of all majority particle states below the Fermi momentum $k_{\mathrm{F}}$, and we use the notation $\sum_{\mathbf{k}>k_{\mathrm{F}}}$ to indicate summation over allowed states.

\section{A. FF and SF bound states}

In the following, we will refer to the many-body polaritonic bound state with finite COM momentum $\left|\Psi_{\mathbf{Q} \neq \mathbf{0}}\right\rangle$ as the FuldeFerrel (FF) state. Note that we use the notation FF rather than FFLO because the pairing wave function we consider is a single plane wave, and thus it does not have any spatial modulation of density [13]. If we would consider increasing the density of minority particles, we expect a smooth evolution from the finite $\mathbf{Q}$ bound state we describe here to a modulated coherent FFLO paired phase [11]. In the absence of cavity photons, the finite $\mathbf{Q}$ bound state for a single impurity has already been analyzed for GaAs [10] and TMDC [12] structures, where it was predicted to occupy a sizable region of the phase diagram. For an imbalanced state of electron-hole bilayers, with a nonvanishing density of minority particles, a FFLO phase was also described in Refs. $[9,11]$.

Also by analogy to the terminology used to describe the states at nonzero minority density, we refer to the zero COM momentum bound state $\left|\Psi_{\mathbf{0}}\right\rangle$ as the superfluid (SF) state. For a finite minority particle density, the SF state is an excitonic condensate where pairing occurs for a balanced fraction of electrons and holes at zero COM momentum (but finite rela- tive momentum), while the excess majority species occupies a Fermi sea around $\mathbf{k}=\mathbf{0}$.

To find which state occurs in the presence of coupling to photons, we minimize $\left\langle\Psi_{\mathbf{Q}}\left|\left(\hat{H}-E_{\mathbf{Q}}-E_{g}\right)\right| \Psi_{\mathbf{Q}}\right\rangle$ with respect to the complex amplitudes $\varphi_{\mathbf{k}}$ and $\alpha_{\mathbf{Q}}$ (16). This gives the coupled eigenvalue equations

$$
\begin{aligned}
\left(E_{\mathbf{Q}}-\xi_{\mathbf{k} \mathbf{Q}}\right) \varphi_{\mathbf{k} \mathbf{Q}} & =-\sum_{\mathbf{k}^{\prime}>k_{\mathrm{F}}} \frac{V_{\mathbf{k}-\mathbf{k}^{\prime}}}{\mathcal{A}} \varphi_{\mathbf{k}^{\prime} \mathbf{Q}}+g \alpha_{\mathbf{Q}}, \\
\left(E_{\mathbf{Q}}-v_{\mathrm{C} \mathbf{Q}}+E_{g}\right) \alpha_{\mathbf{Q}} & =\frac{g}{\mathcal{A}} \sum_{\mathbf{k}>k_{\mathrm{F}}} \varphi_{\mathbf{k} \mathbf{Q}} .
\end{aligned}
$$

The lowest energy eigenvalue $E_{\mathbf{Q}}$ represents the energy of a bound lower polariton state in the presence of a Fermi sea, accounting for the modification of the exciton wave function both by light-matter coupling and by Pauli blocking. Here, $\xi_{\mathbf{k} \mathbf{Q}}=\epsilon_{\mathbf{Q}-\mathbf{k}, 2}+\epsilon_{\mathbf{k}, 1}-\frac{1}{\mathcal{A}} \sum_{\mathbf{k}^{\prime}<k_{\mathrm{F}}} U_{\mathbf{k}-\mathbf{k}^{\prime}}$ includes the exchange correction to the electron dispersion. Note again that we define the energy $E_{\mathbf{Q}}$ with respect to the band-gap energy $E_{g}$; furthermore, we neglect the energy of the interacting Fermi sea $|\mathrm{FS}\rangle, \quad \mathscr{E}_{\mathrm{FS}}=\sum_{\mathbf{k}<k_{\mathrm{F}}}\left[\epsilon_{\mathbf{k}, 1}+E_{g} / 2-\sum_{\mathbf{k}^{\prime}<k_{\mathrm{F}}} U_{\mathbf{k}-\mathbf{k}^{\prime}} /(2 \mathcal{A})\right]$, because we are interested in comparing $E_{\mathbf{Q}}$ with that of the normal state, which also includes $\mathscr{E}_{\mathrm{FS}}$ (see Sec. III B).

In the absence of photons, we set $g=0$ in Eq. (17a) and obtain the energy $E_{\mathbf{Q}}=E_{\mathrm{X} \mathbf{F}}^{\left(d, E_{\mathrm{F}}\right)}$ of a many-body exciton state in the presence of a Fermi sea as the lowest energy solution of the Schrödinger equation,

$$
\left(E_{\mathbf{Q}}-\xi_{\mathbf{k} \mathbf{Q}}\right) \varphi_{\mathbf{k} \mathbf{Q}}=-\sum_{\mathbf{k}^{\prime}>k_{\mathrm{F}}} \frac{V_{\mathbf{k}-\mathbf{k}^{\prime}}}{\mathcal{A}} \varphi_{\mathbf{k}^{\prime} \mathbf{Q}}
$$

At zero doping and for a single layer, $E_{\mathrm{X} \mathbf{0}}^{\left(d=0, E_{\mathrm{F}}=0\right)}=E_{\mathrm{X}}^{(d=0)}=$ $-R_{\mathrm{X}}<0$ as given in Eq. (5). The eigenvalue problem in Eq. (18) has been solved numerically for GaAs electron-hole structures in Ref. [10]. There, it was found that, when increasing the majority particle density, the many-body excitonic state eventually acquires a finite COM momentum $\mathbf{Q}$, as this state reduces the kinetic energy cost. As such, this FF-like state induced by Pauli blocking is favored when the minority particle is lighter. Further, as also discussed below, the longrange nature of the Coulomb interaction also stabilizes the finite $\mathbf{Q}$ exciton state [10]. Recently, these results have been extended to the specific case of TMDC monolayers [12]. Note that, in the presence of a Fermi sea, one cannot just consider the sign of $E_{\mathrm{XQ}}^{\left(d, E_{\mathrm{F}}\right)}$ to determine whether the manybody exciton state is bound or not. One must instead compare $E_{\mathrm{XQ}}^{\left(d, E_{\mathrm{F}}\right)}$ with the energy of the normal state, as defined next.

\section{B. Normal state}

Under some conditions, we find that at large majority particle density, the finite COM momentum exciton can undergo an unbinding transition to the normal $(\mathrm{N})$ state. This comprises an unbound minority particle on top of a Fermi sea of majority particles:

$$
\left|\Psi_{\mathrm{N}}\right\rangle=\hat{c}_{\mathbf{0}, 2}^{\dagger} \hat{c}_{k_{\mathrm{F}} \hat{\mathbf{k}}, 1}^{\dagger}|\mathrm{FS}\rangle
$$


(a)

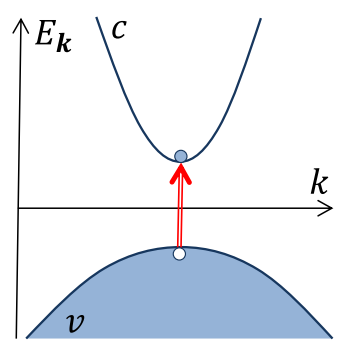

(b)

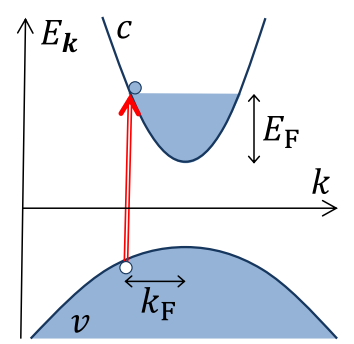

FIG. 2. Particle-hole excitation process via a photon without (a) and with (b) a Fermi sea-all photon-mediated transitions are approximately vertical in a cavity.

where $\hat{\mathbf{k}}$ is an arbitrary direction, and this state has energy

$$
E_{\mathrm{N}}=E_{\mathrm{F}}-\frac{1}{\mathcal{A}} \sum_{\mathbf{k}^{\prime}<k_{\mathrm{F}}} U_{k_{\mathrm{F}} \hat{\mathbf{k}}-\mathbf{k}^{\prime}},
$$

where, as for $E_{\mathbf{Q}}$, we are defining this with respect to $E_{g}$ and neglecting the energy of the interacting Fermi sea, $\mathscr{E}_{\mathrm{FS}}$.

In the absence of light-matter coupling, $g=0$, the excitonic FF state $\left|\Psi_{\mathbf{Q}}\right\rangle$ (16) would reduce to the normal state $\left|\Psi_{\mathrm{N}}\right\rangle$ when we take $\mathbf{Q}=k_{\mathrm{F}} \hat{\mathbf{k}}$ and the exciton wave function takes the form $\varphi_{\mathbf{k} \mathbf{Q}}=\sqrt{\mathcal{A}} \delta_{\mathbf{k}, k_{\mathrm{F}} \hat{\mathbf{k}}}$. This corresponds to a wave function that has weight only when relative and COM momenta are equal and match the Fermi momentum $k_{\mathrm{F}}$.

In Ref. [10] it was shown that unscreened Coulomb interactions $\left(N_{s}=0\right)$ always lead to a bound many-body exciton state for any value of the density, and thus the normal state (19) is never the ground state. We will show here that this is the case also in the presence of light-matter coupling. When screening is nonzero, however, a normal state can occur. It is worth noting that when this state occurs, the only possible normal state is purely electronic-i.e., it has zero photon fraction and is thus given by Eq. (19). This can be seen from the renormalization scheme of the photon energy (11), which has the consequence that any nonzero photon fraction always implies a bound state between minority and majority particles. That is to say, the presence of light can bind an otherwise unbound electron-hole pair.

\section{Effective photon energy in the presence of a Fermi gas}

To understand how the ground state evolves with doping, it is instructive to consider how the effective photon energy changes as the majority density increases, due to a modification of the dielectric constant of the quantum well. As described in Sec. II A, in order to reproduce the experimental protocol for measurements, we have defined the renormalization of the photon energy using a procedure defined at zero gating/doping $E_{\mathrm{F}}=0$. This means that we define the renormalized photon energy $\omega_{\mathrm{C} 0}$ (or equivalently the photonexciton detuning $\delta$ ) in such a way that it approximately matches what would be experimentally measured at $E_{\mathrm{F}}=0$. As illustrated in Fig. 2, the available particle-hole excitations contributing to the dressing of the photon depend on $E_{\mathrm{F}}$. As such, at a finite density of majority species, the effective photon energy $\omega_{\mathrm{CQ}}^{\left(E_{\mathrm{F}}\right)}$ differs from $\omega_{\mathrm{CQ}}$ defined at $E_{\mathrm{F}}=0$. Here, we want to identify and estimate the magnitude of the photon energy shift in the presence of doping. We start by rewriting the eigenvalue equations (17a) and (17b) in an equivalent form by inserting Eq. (17a) in (17b) and defining the new wave function $\beta_{\mathbf{k} \mathbf{Q}}=\frac{1}{\mathcal{A}} \sum_{\mathbf{k}^{\prime}>k_{\mathrm{F}}} V_{\mathbf{k}-\mathbf{k}^{\prime}} \varphi_{\mathbf{k}^{\prime} \mathbf{Q}} /\left(-E_{\mathbf{Q}}+\xi_{\mathbf{k} \mathbf{Q}}\right)$ :

$$
\left(E_{\mathbf{Q}}-v_{\mathrm{C} \mathbf{Q}}+E_{g}+\frac{g^{2}}{\mathcal{A}} \sum_{\mathbf{k}>k_{\mathrm{F}}} \frac{1}{-E_{\mathbf{Q}}+\xi_{\mathbf{k} \mathbf{Q}}}\right) \alpha_{\mathbf{Q}}=\frac{g}{\mathcal{A}} \sum_{\mathbf{k}>k_{\mathrm{F}}} \beta_{\mathbf{k} \mathbf{Q}} .
$$

The divergence of the sum on the left-hand side of Eq. (21) is exactly canceled by the renormalization of the bare photon energy $v_{\mathrm{CQ}}$ by particle-hole excitations, as described in Sec. II A. The form of Eq. (21) suggests that, in the presence of a Fermi sea, the effective renormalized photon energy can be estimated as

$$
\omega_{\mathrm{CQ}}^{\left(E_{\mathrm{F}}\right)} \simeq v_{\mathrm{CQ}}-\frac{g^{2}}{\mathcal{A}} \sum_{\mathbf{k}>k_{\mathrm{F}}} \frac{1}{-E_{\mathbf{Q}}+\xi_{\mathbf{k Q}}} .
$$

This estimate is expected to be valid in the limit of small lightmatter coupling and sufficiently small density, where there is a well-defined exciton bound state that is only weakly perturbed by light. In this limit, one can approximate $E_{\mathbf{Q}} \simeq E_{\mathrm{XQ}}^{\left(d, E_{\mathrm{F}}\right)}$. Taking the COM momentum to be zero, we then estimate the difference between $\omega_{\mathrm{CO}}^{\left(E_{\mathrm{F}}\right)}$ and the photon energy $\omega_{\mathrm{C} 0}$ at zero doping (11) as

$$
\begin{aligned}
\omega_{\mathrm{C} \mathbf{0}}^{\left(E_{\mathrm{F}}\right)}-\omega_{\mathrm{C} \mathbf{0}} \simeq & -\frac{g^{2}}{\mathcal{A}} \sum_{\mathbf{k}>k_{\mathrm{F}}} \frac{1}{-E_{\mathrm{X} \mathbf{0}}^{\left(d, E_{\mathrm{F}}\right)}+\xi_{\mathbf{k} \mathbf{0}}} \\
& +\frac{g^{2}}{\mathcal{A}} \sum_{\mathbf{k}} \frac{1}{-E_{\mathrm{X}}^{(d)}+\epsilon_{\mathbf{k}, 1}+\epsilon_{\mathbf{k}, 2}} .
\end{aligned}
$$

This energy difference is clearly finite because the logarithmic divergence of the first sum cancels with that of the second sum. Thus, we see that the photon energy shift with doping depends quadratically on the light-matter coupling strength $g$, provided $\Omega \ll\left|E_{\mathrm{X} \mathbf{0}}^{\left(d, E_{\mathrm{F}}\right)}-E_{\mathrm{N}}\right|$. By numerically evaluating the density dependence of the exciton energy at $\mathbf{Q}=\mathbf{0}, E_{\mathrm{X} \mathbf{0}}^{\left(d, E_{\mathrm{F}}\right)}$ (see Appendix B), as well as the exchange correction to the electron dispersion, we find that, in the small $\Omega$ and $E_{\mathrm{F}}$ limit, the photon energy shift $\omega_{\mathrm{C} 0}^{\left(E_{\mathrm{F}}\right)}-\omega_{\mathrm{C} 0}$ is always negative (see the solid line of Fig. 3). Such a shift could be observed in experiments either by comparing structures with different Rabi splittings or by changing the doping.

An alternative way of estimating the photon energy shift $\omega_{\mathrm{C} \mathbf{F}}^{\left(E_{\mathrm{F}}\right)}-\omega_{\mathrm{C} \boldsymbol{0}}$ in the presence of a Fermi sea is by identifying the detuning $\delta_{50 \%}$ at which the many-body $\mathbf{Q}=\mathbf{0}$ exciton state and the cavity photon are at resonance:

$$
\omega_{\mathrm{C} \boldsymbol{F}}^{\left(E_{\mathrm{F}}\right)}=E_{\mathrm{X} \boldsymbol{0}}^{\left(d, E_{\mathrm{F}}\right)}+E_{g} .
$$

We assume that this condition is satisfied when the photon fraction $\left|\alpha_{0}\right|^{2}$ is $1 / 2$. We can rewrite the condition (24), which defines the detuning at resonance, $\delta_{50 \%}$, by subtracting the energy of the photon mode at zero doping/gating $\omega_{\mathrm{C} 0}$ (11) from both sides. Then using the definition $\delta=\omega_{\mathrm{C} 0}-\left(E_{\mathrm{X}}^{(d)}+\right.$ 

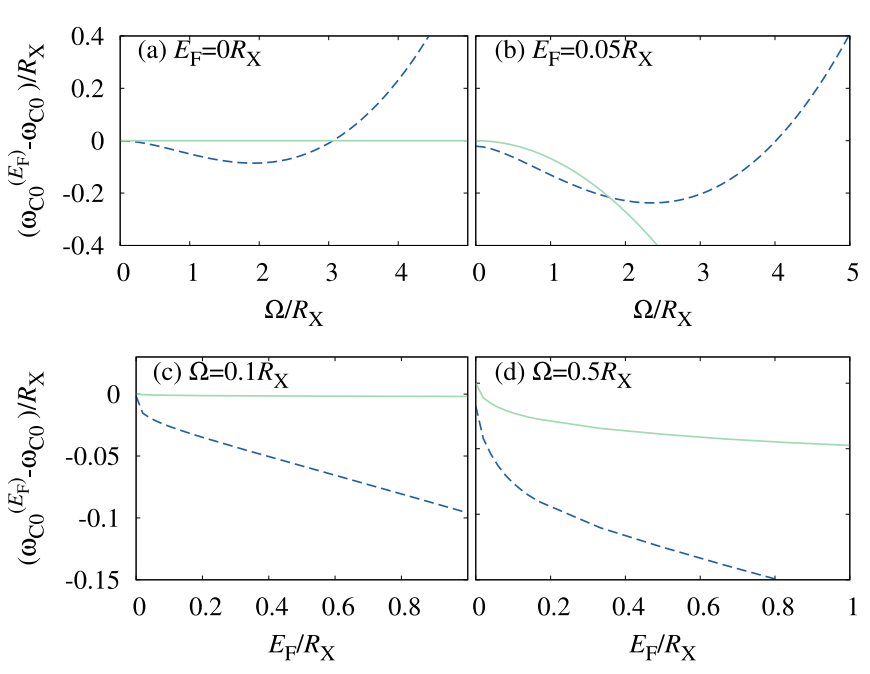

FIG. 3. Photon energy shift in the presence of a Fermi gas $\omega_{\mathrm{C} 0}^{\left(E_{\mathrm{F}}\right)}-\omega_{\mathrm{C} 0}$ as estimated from Eq. (23) (solid line) and from Eq. (25) (dashed line) for either fixed Fermi energy $E_{\mathrm{F}}$ and varying Rabi splitting $\Omega$ (a),(b) or conversely fixed $\Omega$ and varying $E_{\mathrm{F}}$ (c),(d). Parameters are for a GaAs single quantum well $(d=0)$, mass ratio $m_{2} / m_{1}=0.25$, and screened interactions $N_{s}=1$.

$\left.E_{g}\right)$ on the right-hand side gives

$$
\omega_{\mathrm{C} \mathbf{F}}^{\left(E_{\mathrm{F}}\right)}-\omega_{\mathrm{C} \mathbf{0}}=E_{\mathrm{X} \mathbf{0}}^{\left(d, E_{\mathrm{F}}\right)}-E_{\mathrm{X}}^{(d)}-\delta_{50 \%} .
$$

We can thus estimate the photon shift $\omega_{\mathrm{CO}}^{\left(E_{\mathrm{F}}\right)}-\omega_{\mathrm{C} \boldsymbol{0}}$ at a fixed value of $E_{\mathrm{F}}$ and $\Omega$ by evaluating $E_{\mathrm{X} \boldsymbol{0}}^{\left(d, E_{\mathrm{F}}\right)}-E_{\mathrm{X}}^{(d)}$, i.e., by solving Eq. (18), and by numerically estimating the value of detuning $\delta_{50 \%}$ at which the photon fraction is exactly $1 / 2$. The results of this estimate are plotted in Fig. 3 and compared with those obtained from Eq. (23). Note that, even at $E_{\mathrm{F}}=0$, this estimate predicts a photon energy shift because, beyond the weak-coupling regime $g \ll a_{\mathrm{X}} R_{\mathrm{X}}$, the exciton wave function is strongly modified by matter-light coupling, affecting the definition of detuning $\delta$ given in Eq. (12) (see the discussion in Appendix A and Fig. 9). At small and finite $E_{\mathrm{F}}$, the estimates given by Eqs. (23) and (25) agree for small $\Omega$ giving a negative shift of the photon energy, while when $\Omega$ increases, Eq. (25) predicts an upturn of the shift to positive values.

Predicting the exact behavior of $\omega_{\mathrm{C} 0}^{\left(E_{\mathrm{F}}\right)}-\omega_{\mathrm{C} 0}$ with either $\Omega$ or $E_{\mathrm{F}}$ is nontrivial, since both estimates of Eqs. (23) and (25) are based on the assumption that the system behaves like a coupled oscillator model, a hypothesis that loses validity when either $\Omega$ or $E_{\mathrm{F}}$ increases. As we will see in the next section, the shift of the photon energy with doping has little consequence for the phase diagram at fixed Rabi splitting $\Omega$, while the implications are larger when we fix $E_{\mathrm{F}}$ and change $\Omega$.

\section{Numerical implementation}

We obtain the ground-state phase diagram by numerically diagonalizing the coupled equations (17) and analyzing the nature of the lowest energy state while comparing it with the energy of the normal state (20). We use a nonlinear grid in the relative momentum $\mathbf{k}$-space and evaluate, at a given value of the COM momentum $\mathbf{Q}$, the lowest eigenvalue $E_{\mathbf{Q}}$ and the associated excitonic $\varphi_{\mathbf{k}}$ and photonic $\alpha_{\mathbf{Q}}$ eigenvectors, with
$\left|\alpha_{\mathbf{Q}}\right|^{2}$ representing the state photon fraction. The results we show are numerically converged with respect to the number of points employed in the momentum grid. We then minimize the energy $E_{\mathbf{Q}}$ with respect to $Q \equiv|\mathbf{Q}|$, and we indicate the momentum at which the energy is minimized by $Q_{\min }$.

In the following, we rescale energies by the $2 \mathrm{D}$ exciton binding energy $R_{\mathrm{X}}$ and lengths by the Bohr radius $a_{\mathrm{X}}$ defined in Eqs. (5). Hence, only a few independent dimensionless parameters are left to characterize the system properties and phase diagram, namely the mass ratio between minority and majority particles, $m_{2} / m_{1}$; the rescaled bilayer distance, $d / a_{\mathrm{X}}$; the dimensionless majority particle density, $E_{\mathrm{F}} / R_{\mathrm{X}}$; the photon-exciton detuning, $\delta / R_{\mathrm{X}}$ [Eq. (12)]; and the Rabi splitting, $\Omega / R_{\mathrm{X}}[$ Eq. (13)].

\section{CHARGE-IMBALANCED QUANTUM WELLS IN PLANAR MICROCAVITIES}

We first consider the case of a GaAs quantum-well system embedded in a microcavity. In Fig. 4 we show our calculated phase diagram as a function of majority particle density and detuning, keeping the Rabi splitting fixed. We compare the results for both screened and unscreened Coulomb interactions, for a single quantum well $(d=0)$ and a bilayer geometry $\left(d=a_{\mathrm{X}}\right)$, and for one electron in a Fermi sea of holes $\left(m_{2} / m_{1}=0.25\right)$ and one hole in a Fermi sea of electrons $\left(m_{2} / m_{1}=4\right)$. In all cases, we see that the coupling to cavity light modes suppresses the formation of the finite momentum FF state as compared to the case without light-matter coupling. In particular, a strong coupling to light favors the $\mathbf{Q}=\mathbf{0}$ state, since the photon mode at nonzero $\mathbf{Q}$ is at high energy, due to the small photon mass. As such, strong coupling to light imposes that for detunings below a minimal value, $\delta<\delta_{\min }$, only the $\mathbf{Q}=\mathbf{0}$ SF phase is allowed.

Fixing the detuning $\delta>\delta_{\min }$ and increasing $E_{\mathrm{F}}$, one first finds a SF-FF transition between a $\mathbf{Q}=\mathbf{0}$ many-body mixed polariton state and a $Q_{\min } \neq 0 \mathrm{FF}$ state weakly coupled to light. This state has also been referred to as a roton minimum [12]. This occurs because the energy gained by forming a finite $\mathbf{Q}$ exciton state is larger than that obtained by dressing the $\mathbf{Q}=\mathbf{0}$ exciton with a zero momentum photon. For screened interactions, the transition can be directly to the unbound $\mathrm{N}$ state, while for unscreened interactions there is no normal phase, just as in the absence of photons [10]. Both the SF-FF and SF-N transitions are first order (see Appendix C), with $Q_{\min }$ changing discontinuously from $Q_{\min }=0$ to a finite value, as shown in Fig. 5. Because of the small cavity photon mass, the finite $Q_{\min }$ FF phase has a small photon fraction that decreases further upon increasing $E_{\mathrm{F}}$ (see Fig. 5). Thus, the value of $Q_{\min }$ almost coincides with that in the absence of the cavity field, and in particular $Q_{\min }$ locks to $k_{\mathrm{F}}$ at the FF-N transition. In contrast, for unscreened interactions, $Q_{\text {min }}$ asymptotically tends to $k_{\mathrm{F}}$ in the FF region only for large values of $E_{\mathrm{F}}$. In addition, the FF-N transition is always second order and it is only weakly affected by the coupling to lightthus it is approximately independent of both $\delta$ and $\Omega$.

The SF-FF transition is strongly affected by the coupling to a cavity field. In particular, the many-body exciton at $\mathbf{Q}=\mathbf{0}$ strongly couples to the cavity photon when both energies are comparable, resulting in a half-matter half-light many-body 

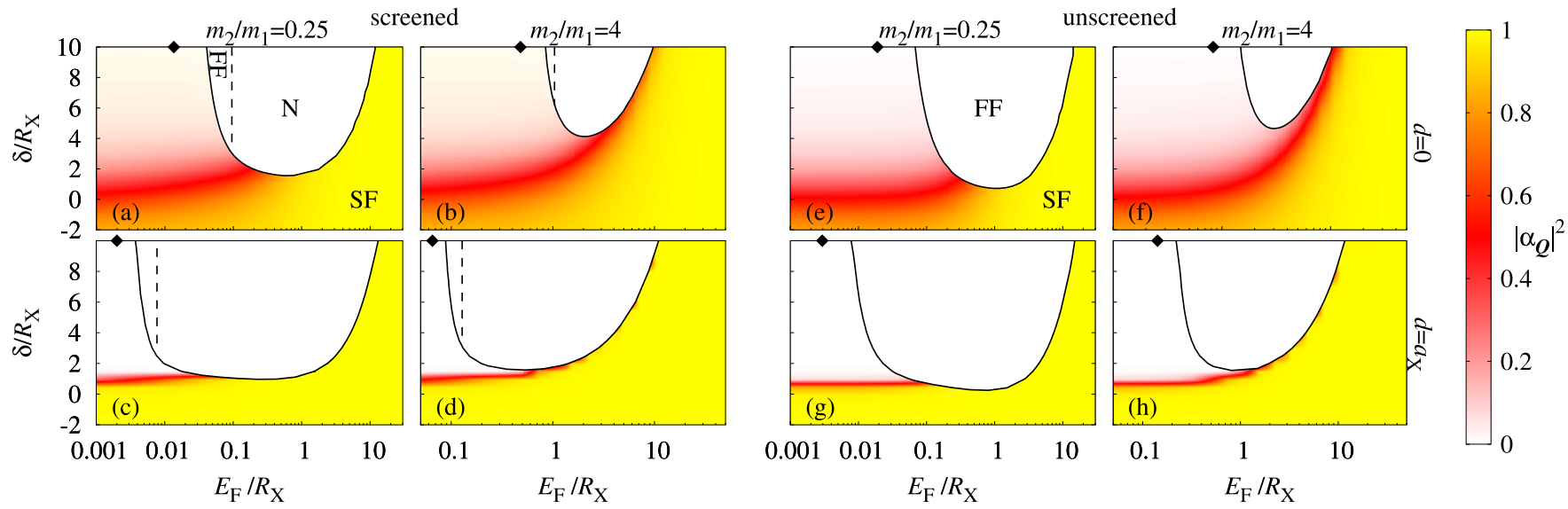

FIG. 4. Phase diagram of photon-exciton detuning $\delta$ and majority particle Fermi energy $E_{\mathrm{F}}$ for a GaAs heterostructure with either a single quantum well ( $d=0$ ) [panels (a),(b),(e),(f)] or a bilayer geometry at a distance $d=a_{\mathrm{X}}$ [(c),(d),(g),(b)]. Panels (a)-(d) are for RPA screened Coulomb interactions $\left(N_{s}=1\right)$, while (e)-(h) are for unscreened interactions $\left(N_{s}=0\right)$. The mass ratio is fixed to either $m_{2} / m_{1}=0.25$, i.e., one electron in a Fermi sea of holes, or $m_{2} / m_{1}=4$, i.e., one hole in a Fermi sea of electrons. The Rabi splitting is fixed to $\Omega=2 R_{\mathrm{X}}$ for the $d=0$ case and to $\Omega=2\left|E_{\mathrm{X}}^{(d)}\right| \simeq 0.64 R_{\mathrm{X}}$ for the bilayer at the $d=a_{\mathrm{X}}$ case. Solid lines are first-order transitions (SF-FF and SF-N). The dashed almost vertical line is the second-order FF-N transition occurring for screened interactions. First- and second-order transitions meet at a critical end point. The diamond symbols indicate the value of the density, $E_{\mathrm{F} 0}$, at which the SF-FF transition occurs in the absence of the cavity field $\Omega=0=\alpha_{\mathbf{Q}}$. The color map represents the photon fraction $\left|\alpha_{\mathbf{Q}}\right|^{2}$.

polariton state. In Fig. 4, the red region of the color map indicates where the photon fraction is around 50\%, corresponding to resonance between the cavity photon and the many-body exciton. The value of the detuning $\delta$ for which resonance occurs is seen to grow with the majority density. This is mostly due to the $\mathbf{Q}=\mathbf{0}$ exciton energy $E_{\mathrm{X} \mathbf{0}}^{\left(d, E_{\mathrm{F}}\right)}$ growing with $E_{\mathrm{F}}$ due to Pauli blocking (see Appendix B). Indeed, one can show that $E_{\mathrm{X} \mathbf{0}}^{\left(d, E_{\mathrm{F}}\right)}$ grows sublinearly for $E_{\mathrm{F}} \ll R_{\mathrm{X}}$ and screened

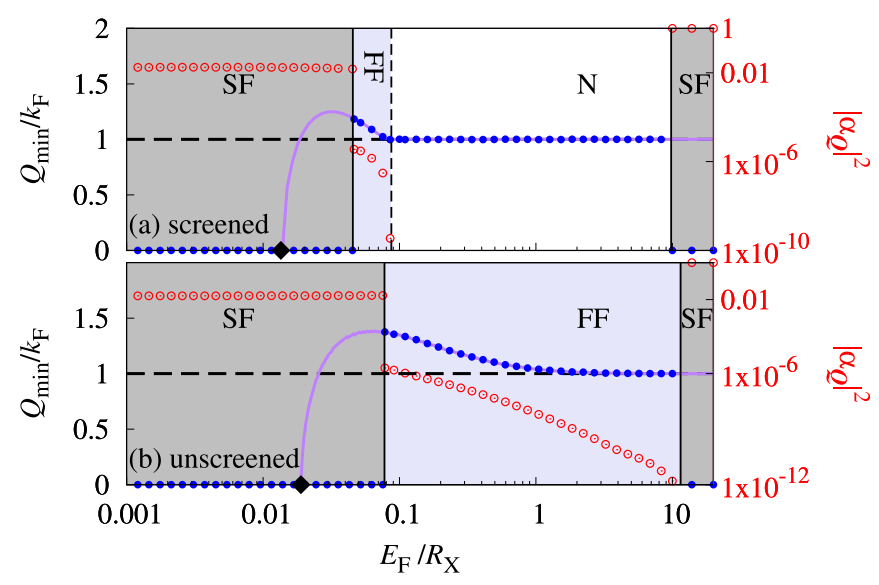

FIG. 5. Momentum $Q_{\min }$ (filled [blue] circles) minimizing the many-body polaritonic energy $E_{\mathbf{Q}}$ as a function of the majority Fermi energy $E_{\mathrm{F}}$ for a single quantum well $d=0$, mass ratio $m_{2} / m_{1}=$ 0.25 , Rabi splitting $\Omega=2 R_{\mathrm{X}}$ and detuning $\delta=8 R_{\mathrm{X}}$. Interactions are RPA screened $\left(N_{s}=1\right)$ in panel (a), and unscreened $\left(N_{s}=0\right)$ in panel (b). Solid (violet) lines represent the value of $Q_{\min }$ in the absence of light-matter coupling $(\Omega=0)$, while the thick dashed (black) line is the Fermi momentum $k_{\mathrm{F}}$. The corresponding photon fraction $\left|\alpha_{\mathbf{Q}}\right|^{2}$ is plotted with open (red) circles and the corresponding axes are on the right side of each panel. interaction, while it grows linearly $\sim E_{\mathrm{F}}$ for $E_{\mathrm{F}}>R_{\mathrm{X}}$ (see Appendix B and Figs. 10 and 11).

At large positive detunings, we recover, as expected, the results obtained in Ref. [10] for GaAs single wells and bilayers in the absence of light-matter coupling. Here, as one increases the majority particle density, Pauli blocking causes the many-body exciton energy $E_{\mathbf{Q}}=E_{\mathrm{XQ}}^{\left(d, E_{\mathrm{F}}\right)}$ obtained by solving Eq. (18) to develop a minimum at finite COM momentum $Q_{\min }$, as this reduces the kinetic energy cost of the minority particle. We denote the Fermi energy at which this transition occurs in the excitonic limit by $E_{\mathrm{F} 0}$, and, in the figures, this is illustrated by a diamond symbol. Without light, the transition to the FF state is always second order (see Appendix C).

By further increasing the density at fixed (large positive) photon-exciton detuning, there is eventually an additional first-order transition to an almost completely photonlike $\mathbf{Q}=$ 0 SF state. This is because the energy of the FF and N states is pushed up by Pauli blocking such that they exceed the photon energy at sufficiently large density. As such, larger values of the detuning require larger values of density for this second transition to occur. Since this transition depends only weakly on the light-matter coupling, the FF-SF (N-SF) boundary essentially occurs when $\delta \simeq E_{\mathrm{X}}^{\left(d, E_{\mathrm{F}}\right)}-E_{\mathrm{X}}^{(d)}\left(\delta \simeq E_{\mathrm{N}}-E_{\mathrm{X}}^{(d)}\right)$, where $E_{\mathrm{XQ}_{\min }}^{\left(d, E_{\mathrm{F}}\right)}$ is the FF many-body exciton energy at Fermi energy $E_{\mathrm{F}}$ and bilayer distance $d$ in the absence of the photon field-see Eq. (18).

From the study of the phase diagram at fixed Rabi splitting, we can draw similar conclusions about the mechanisms promoting the existence of a FF phase to those known in the absence of the cavity photon [10]: the FF phase is favored by unscreened Coulomb interactions and by a small minority particle mass. In addition, considering the unscreened case, a finite bilayer distance also favors FF. This is because the interlayer interaction suppresses large momentum scattering 


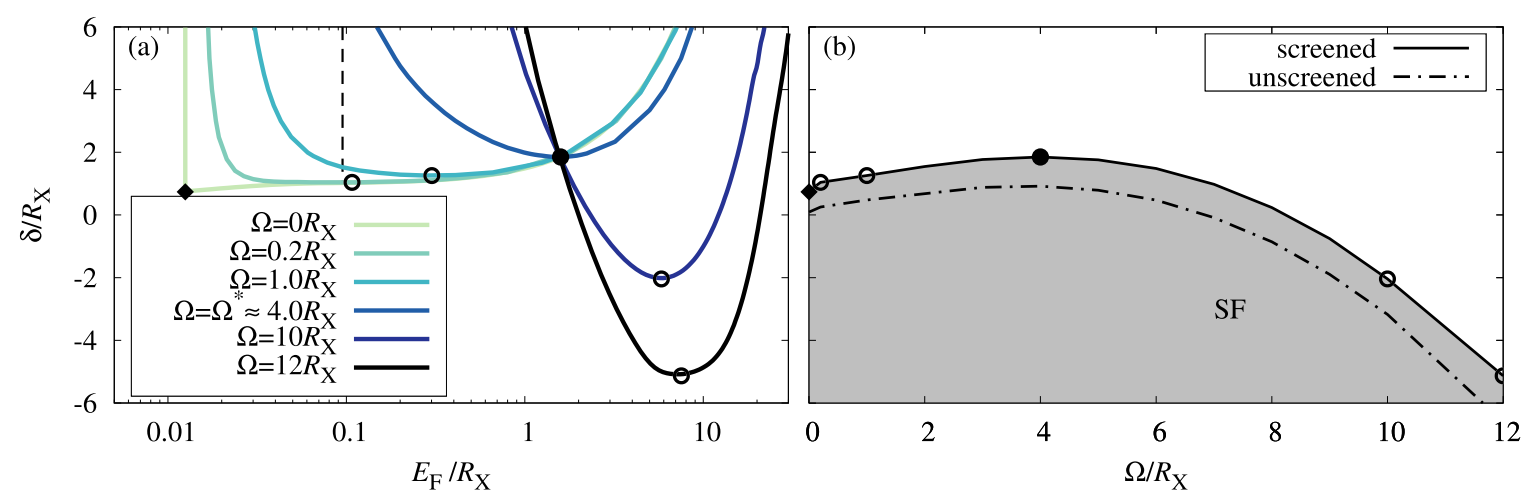

FIG. 6. In panel (a), the solid lines are SF-FF (or SF-N) phase boundaries for different values of the Rabi splitting $\Omega$, for a single quantum well with hole doping, $d=0$ and $m_{2} / m_{1}=0.25$, and for screened interactions $N_{s}=1$. In particular, the region above a solid line is either FF (on the left of the dashed line) or N (on the right). The almost vertical dashed line is the approximately $\Omega$-independent FF-N boundary (see Fig. 4). Below each solid line, the phase is SF. Each symbol represents the minimal detunings $\delta_{\min }$ of the boundaries-special values are $\Omega=0$ (filled diamond) and the $\Omega$ at which $\delta_{\min }=\delta^{*}$ (filled circle). A special value common to all boundaries is ( $\left.\delta^{*}, E_{\mathrm{F}}^{*}\right)$ (filled circle). In panel (b), the solid line and symbols give the behavior of $\delta_{\min }$ as a function of $\Omega$ for screened $N_{s}=1$ interactions, while the dot-dashed line represents $\delta_{\min }$ for unscreened $N_{s}=0$ interactions.

and promotes an exciton wave function $\varphi_{\mathbf{k}} \mathbf{Q}$ peaked at the $\mathbf{k} \sim \mathbf{Q}$ direction, and also because a finite interlayer distance reduces the effective electron-hole coupling to light. While our results demonstrate that embedding the quantum-well structure into a cavity reduces the parameter region where FF can occur, this phase is still weakly coupled to light. Thus, the FF ground state should be visible in the photon momentum distribution, in an experiment with sufficient sensitivity. Note that for our simplified scenario in Eq. (16) of a single minority particle and thus a single photon in the cavity, the system photoluminescence is peaked at the energy $E_{\mathbf{Q}}+E_{g}$, with a weight given by the corresponding photon fraction $\left|\alpha_{\mathbf{Q}}\right|^{2}$. Because, as shown in Fig. 5, this photon fraction is very small, it would require a very sensitive experimental probe. Even when (in the presence of strong coupling to light) the lowest energy state is a $\mathbf{Q}=\mathbf{0}$ polariton state, it has been suggested that the formation of a FF state could drastically change photoluminescence, due to the bottleneck effect of high momentum excitons relaxing to the true lowest energy polariton state [45].

\section{A. Comparison of structures with different Rabi splitting}

It is possible to study the evolution of the FF phase with changing Rabi splitting by considering a sequence of cavities that have different numbers $N_{\mathrm{QW}}$ of embedded quantum wells, since $\Omega \sim \sqrt{N_{\mathrm{QW}}}[46,47]$. In particular, in Ref. [44], two structures with either 1 or 28 quantum wells stacked at the antinodes of the cavity field have been compared, allowing one to study the change of the Rabi splitting in the range $0.3 R_{\mathrm{X}} \lesssim \Omega \lesssim 1.3 R_{\mathrm{X}}$. Note that in inorganic microcavities, while typically $\Omega \ll E_{g}$, the very-strong-coupling regime $\Omega>R_{\mathrm{X}}$ can also be routinely achieved [47-49]. Studying the evolution of the phase diagram with increasing Rabi splitting should in principle directly show how the introduction of light-matter coupling modifies the phase diagram.

With this motivation, in Fig. 6(a), we compare the boundaries between the SF and the FF ( $\mathrm{SF}$ and $\mathrm{N}$ ) phases for different values of $\Omega$. Screened and unscreened interactions give qualitatively the same results, with the only difference being the absence of the $\mathrm{N}$ phase for unscreened interactions. The boundaries are also quantitatively similar in the two cases. In the absence of light-matter coupling, the SF-FF boundary is given by $\left(E_{\mathrm{F}}>E_{\mathrm{F} 0}\right)$

$$
\delta=E_{\mathrm{X} \mathbf{Q}_{\min }}^{\left(d, E_{\mathrm{F}}\right)}-E_{\mathrm{X}}^{(d)},
$$

where $E_{\mathrm{X}}^{\left(d, E_{\mathrm{F}}\right)}$ is the FF many-body exciton energy at Fermi energy $E_{\mathrm{F}}$. For the SF-N boundary at $\Omega=0$, this expression becomes $\delta=E_{\mathrm{N}}-E_{\mathrm{X}}^{(d)}$. We observe an evolution of the minimal photon-exciton detuning $\delta_{\min }$ with $\Omega$ [Fig. 6(b)], which, starting from the value $\delta_{0}=E_{\mathrm{X}}^{\left(d, E_{\mathrm{F} 0}\right)}-E_{\mathrm{X}}^{(d)}$ at $\Omega=0$, grows up to a maximum value $\delta^{*}$, and then decreases again. Consequently, the light-matter coupling is detrimental to the formation of a finite momentum phase for small values of $\Omega$, while it favors finite $\mathbf{Q}$ at $\Omega \gtrsim 4 R_{\mathrm{X}}$.

There is a special point $\left(\delta^{*}, E_{\mathrm{F}}^{*}\right)$ that is common to all SF-FF (SF-N) boundaries as one varies $\Omega$, i.e., one observes in Fig. 6(a) that all lines appear to cross at a single point. At this particular value of the photon-exciton detuning and density, all the dependence on the Rabi splitting and thus the light-matter coupling is lost. Here, the decrease in energy due to forming a polariton is exactly counterbalanced by dopinginduced changes to the cavity dielectric constant discussed in Sec. III C. Note that this behavior is not accurately captured by the estimated photon shift in Eq. (23), since this is not valid in the regime $E_{\mathrm{F}}>R_{\mathrm{X}}$. However, we can determine $\left(\delta^{*}, E_{\mathrm{F}}^{*}\right)$ once we account for all the electron-hole scattering processes, as shown in Appendix D. We have checked that the existence of the special point $\left(\delta^{*}, E_{\mathrm{F}}^{*}\right)$ is common to both structures with single-well and bilayer geometry, and it is also independent of whether interactions are screened or unscreened.

To further illustrate the special role played by the detuning $\delta^{*}$ and Fermi energy $E_{\mathrm{F}}^{*}$, we plot in Fig. 7 the three different types of phase diagrams at fixed detuning $\delta$ that arise by varying $\Omega$ and $E_{\mathrm{F}}$. A common feature for all three cases is that, for $E_{\mathrm{F}}<E_{\mathrm{F}}^{*}$, the $\mathrm{FF}$ and $\mathrm{N}$ phases are suppressed upon increasing $\Omega$ in favor of a strongly mixed light-matter polaritonic SF 


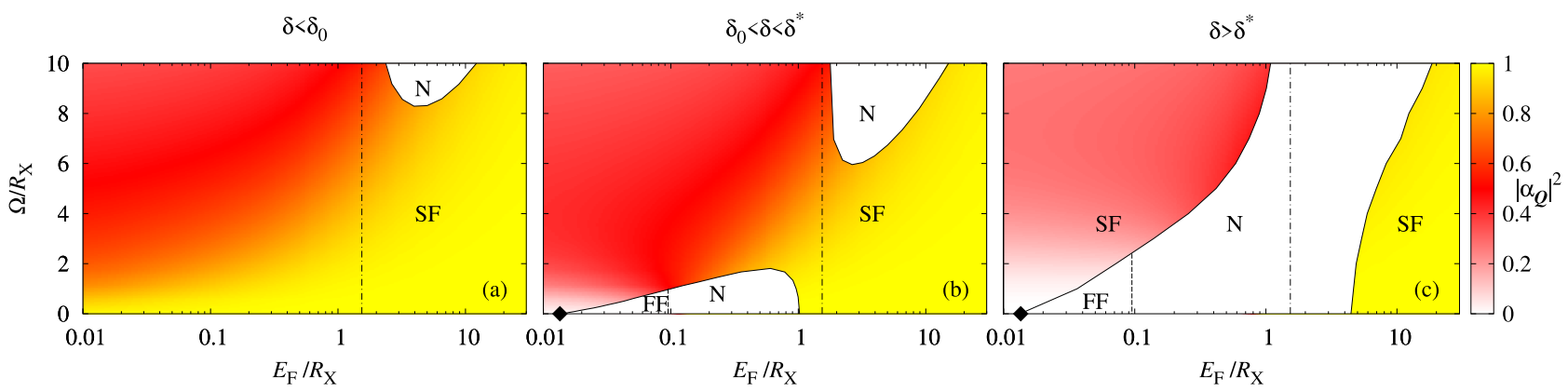

FIG. 7. Different possible topologies of the phase diagram as a function of the Rabi splitting $\Omega$ and the majority particle Fermi energy $E_{\mathrm{F}}$ for a GaAs heterostructure with a single quantum well $(d=0), m_{2} / m_{1}=0.25$, and screened interactions $N_{s}=1$. The detuning has been fixed to $\delta=0<\delta_{0}$ [panel (a)], $\delta_{0}<\delta=1.5 R_{\mathrm{X}}<\delta^{*}$ [panel (b)], and $\delta=4 R_{\mathrm{X}}>\delta^{*}$ [panel (c)]. In all panels, the vertical dot-dashed line is the value of $E_{\mathrm{F}}^{*}$ (see Fig. 6), while all other lines and labels are as in Fig. 4.

phase with $\left|\alpha_{\mathbf{0}}\right|^{2} \sim 0.5$. Note also that for $E_{\mathrm{F}}<E_{\mathrm{F}}^{*}$, the FF (N) phase occurs only for $\delta>\delta_{0}$. In this small $E_{\mathrm{F}}$ case, the lowering of energy of the strongly mixed $\mathbf{Q}=\mathbf{0}$ LP state with $\Omega$ dominates over any change of the cavity dielectric constant because of gating/doping. Note that the phase diagram we see in this small $E_{\mathrm{F}}$ case illustrates the idea that increasing light-matter coupling can stabilize a polaritonic ground state even when the purely excitonic system is unbound.

For $E_{\mathrm{F}}>E_{\mathrm{F}}^{*}$, we see quite a different behavior-a finite momentum $\mathrm{FF}$ or $\mathrm{N}$ phase is favored at larger values of the Rabi splitting $\Omega$, regardless of the value of the detuning. In this large $E_{\mathrm{F}}$ case, the SF-FF (SF-N) transition typically occurs from an almost purely photonic SF phase $\left|\alpha_{0}\right|^{2} \sim 1$ to an almost purely excitonic FF (N) phase with $\left|\alpha_{0}\right|^{2} \ll 1$ $\left(\left|\alpha_{0}\right|^{2}=0\right)$. This transition occurs because the shift in the cavity dielectric constant at finite $E_{\mathrm{F}}$ increases with $\Omega$, while the excitonic or normal state energy is $\Omega$-independent, so that eventually, increasing $\Omega$ to large enough values, one favors the excitonic phase over the polaritonic.

Note that for GaAs heterostructures with a single quantum well and $m_{2} / m_{1}=0.25$, we find that $E_{\mathrm{F}}^{*} \simeq 1.55 R_{\mathrm{X}}\left(E_{\mathrm{F}}^{*} \simeq\right.$ $1.95 R_{\mathrm{X}}$ ) for screened $N_{s}=1$ (unscreened $N_{s}=0$ ) interactions, respectively — see Appendix D. This value of the Fermi energy is well below typical energies at which band curvature and structure start being important, so it lies within the range of the validity of our model. Indeed, from the GaAs lattice constant $a \simeq 0.56 \mathrm{~nm}$, we can estimate that $1 /\left(2 \mu a^{2}\right) \simeq$ $150 R_{\mathrm{X}} \gg E_{\mathrm{F}}^{*}$.

\section{TMDC MONOLAYER EMBEDDED INTO A PLANAR MICROCAVITY}

As mentioned in the Introduction, one context in which electronically doped polariton systems have been studied experimentally are TMDC materials [7,32-34]. We derive here the phase diagram for the specific case of doped $\mathrm{MoSe}_{2}$; see Fig. 8. In particular, we consider the case of a single hole in a Fermi sea of electrons, with all electrons being spin- and valley-polarized, a regime that can be experimentally realized by applying a magnetic field [50]. Further, we have assumed a large enough spin-orbit splitting so that only the lowest energy conduction band is considered.
Due to the fact that most of the dielectric screening takes place within the two-dimensional layer, TMDC materials require a separate analysis from the case of III-V semiconductor heterostructures. Specifically, we consider the same model Hamiltonian as before, Eq. (1), with a screened electron-hole interaction appropriate for a monolayer in vacuum [51-53]:

$$
V_{\mathbf{q}}^{\mathrm{RK}}=\frac{2 \pi e^{2}}{q} \frac{1}{\left(1+r_{0} q\right)} .
$$

For $\mathrm{MoSe}_{2}$, the screening length is $r_{0}=5 \mathrm{~nm}$ [27]. Note that, in contrast to Thomas-Fermi screening, the dielectric screening vanishes at large distances, i.e., $V_{\mathbf{q}}^{\mathrm{RK}} \rightarrow 2 \pi e^{2} / q$ for $q \rightarrow 0$. The electron and hole masses are $m_{1} \equiv m_{e}=0.56 m_{0}$ and $m_{2} \equiv m_{h}=0.59 m_{0}$ [27,54], where $m_{0}$ is the free-electron mass. Because $m_{e}$ and $m_{h}$ have very similar values, little difference is expected whether the minority species is a holeas explicitly considered here-or an electron. Following Ref. [12], we neglect electron exchange; furthermore, we neglect screening by the electron gas on the basis that, for these

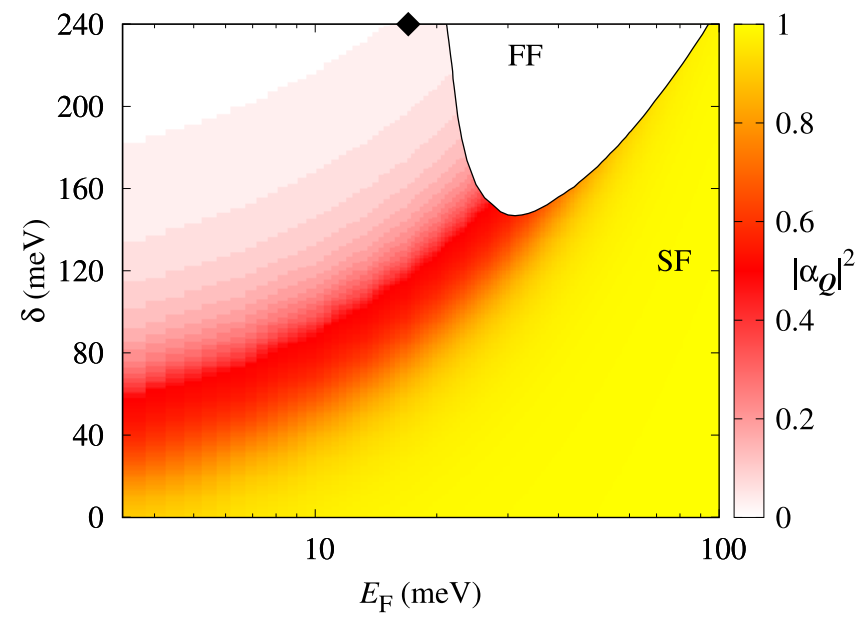

FIG. 8. Phase diagram for a $\mathrm{MoSe}_{2}$ monolayer embedded into a planar cavity as a function of photon-exciton detuning $\delta$ and electron Fermi energy $E_{\mathrm{F}}$. We take the Rabi splitting $\Omega=40 \mathrm{meV}$. The solid line is the first-order SF-FF transition, while the diamond symbol indicates the value of $E_{\mathrm{F} 0}$ at which the SF-FF transition occurs in the absence of the cavity field $\left(\Omega=0=\alpha_{\mathbf{Q}}\right)$ [12]. The color map represents the photon fraction $\left|\alpha_{\mathbf{Q}}\right|^{2}$. 
materials, the plasma frequency, $\omega_{\mathrm{pl}}\left(k_{\mathrm{F}}\right) \simeq \sqrt{n_{1} k_{\mathrm{F}}^{2} V_{k_{\mathrm{F}}}^{\mathrm{RK}} / m_{1}} \sim$ $90 \mathrm{meV}$ (for $E_{\mathrm{F}}=20 \mathrm{meV}$ ), is much smaller that the exciton binding energy $\left|E_{\mathrm{X}}\right|=485 \mathrm{meV}[27,54,55]$.

For TMDC monolayers, strong coupling to light can be attained by placing flakes of material in planar cavities. Strong light-matter coupling leading to exciton-polariton formation is now routinely achieved [31-33,56,57]. We fix the cavity photon mass to $m_{\mathrm{C}}=10^{-5} m_{0}$ and the Rabi splitting to $\Omega=$ $40 \mathrm{meV}$ [31]. Note that, even with such a large value of $\Omega$, because the exciton binding energy is even larger, the regime of very strong coupling [58] $\Omega \gtrsim\left|E_{\mathrm{X}}\right|$ has not yet been reached for TMDCs. However, recently there has been strong progress in this direction; see, e.g., Refs. [59,60]. Importantly for our analysis, the renormalization scheme of the photon energy described in Sec. II A is unchanged.

By considering the same variational many-body polariton state as in Eq. (16), we derive the phase diagram versus detuning $\delta$ and electron Fermi energy $E_{\mathrm{F}}$. The resulting phase diagram is shown in Fig. 8, and is seen to qualitatively agree with the unscreened case of GaAs presented in Fig. 4. Because the long-range unscreened Coulomb interaction promotes the finite momentum bound FF phase, it is not surprising that the system never transitions to the normal state $\mathrm{N}$ for the potential in Eq. (27). As shown in Ref. [10], the bare Coulomb interaction always implies a bound exciton state for any density of majority particles. In the absence of the cavity photon mode, we recover the results of Ref. [12], which predicted a SF-FF transition at $E_{\mathrm{F} 0}=20 \mathrm{meV}$ - as before, this value is labeled with a diamond symbol in Fig. 8. Because of the large value of $\left|E_{\mathrm{X}}\right|$ relative to $\Omega$, the minimal photonexciton detuning for observing $\mathrm{FF}$ is found to be rather large, $\delta_{\text {min }} \simeq 147 \mathrm{meV}$. However, we expect this value to eventually decrease for $\Omega \gtrsim\left|E_{X}\right|$ in a manner similar to that shown in Fig. 6.

\section{CONCLUSIONS AND PERSPECTIVES}

We have studied polaritonic phases in an extremely chargeimbalanced electron-hole mixture in either a single quantum well, a bilayer, or for TMDC monolayers embedded into a planar cavity. In particular, we have analyzed the competition between the formation of an FF-like [13] bound excitonic pair at finite COM momentum, which is promoted by both long-range Coulomb interactions and the Pauli blocking of the Fermi sea $[10,12]$, and the formation of a strongly coupled many-body polariton state at zero momentum, which is promoted by the strong coupling to the cavity field. By fixing the light-matter coupling, i.e., the Rabi splitting, we find that, as expected, strong coupling to a cavity photon mode competes against the formation of the finite momentum FF state, and so reduces the parameter range of majority species density where this phase occurs. Note that the FF phase does weakly couple to light in order to allow its detection in photoluminescence experiments with enough sensitivity. For large photon-exciton detunings, the photon becomes less relevant, and so the FF phase occupies a sizable region at finite density of the majority species. At small densities the FF phase is replaced by bound polariton states with zero COM momentum, which lowers their energy through strong light-matter coupling. At large densities, one instead finds an almost purely photonic state (with zero momentum) because, due to Pauli blocking, the exciton energy grows roughly linearly with the density. As already known for the case without photons, a bound state always exists for unscreened Coulomb interactions, whereas with screening, an unbound state can replace the excitonic FF state.

To understand the topology of the phase diagram, we note that it is important that the presence of a Fermi sea not only changes the energy of the exciton but also the background cavity dielectric constant of the active medium, i.e., the gated/doped quantum well, the bilayer, or the TMDC monolayer. This change has few consequences for the phase diagram at fixed Rabi splitting because the exciton energy shift with density dominates over the shift of the photon energy. However, the photon energy shift increases for sufficiently large values of the Rabi splitting, and consequently it does have a significant effect on the phase diagram at fixed detuning. In particular, we find that increasing the Rabi splitting at low enough doping/gating densities always promotes the formation of a zero momentum strongly bound polariton state. However, surprisingly, at large enough densities, this behavior is reversed, and increasing the coupling to light promotes the formation of finite momentum excitonic states weakly mixed to light.

The results in this paper focus entirely on the regime of extreme imbalance, where there is only a single minority species particle. It is of course interesting to consider the behavior of the many-body state with a larger minority particle density; this will be discussed in a subsequent paper [61]. Another important question concerns the possibility of more complex pairing states, even in the extreme imbalance state. The ansatz we use in this paper assumes that the pairing state has no effect on the majority Fermi sea, however Coulomb interactions between majority particles mean this assumption will not necessarily hold. Relaxing this assumption allows the excitonic state to be dressed by electron-hole pairs of the majority band - such effects have been considered recently for a tightly bound exciton in doped TMDCs [7,40]. Understanding the interplay of this dressing with the internal structure of pairing, the coupling to light, and the crossover from the behavior we discuss here to the Fermi-edge polariton regime is a topic for future work.

The research data underpinning this publication can be accessed at Ref. [62].

\section{ACKNOWLEDGMENTS}

We acknowledge useful discussions with A. Imamoğlu. A.T. and F.M.M. acknowledge financial support from the Ministerio de Economía y Competitividad (MINECO), Project No. MAT2017-83772-R. J.L. and M.M.P. acknowledge support from the Australian Research Council Centre of Excellence in Future Low-Energy Electronics Technologies (CE170100039). J.L. is also supported through the Australian Research Council Future Fellowship FT160100244. A.H.M. and J.K. acknowledge financial support from a Royal Society International Exchange Award, IES \R2\170213. J.K. acknowledges financial support from EPSRC program "Hybrid Polaritonics" (EP/M025330/1). A.H.M. acknowledges support from Army Research Office (ARO) Grant No. W911NF17-1-0312 (MURI) by Welch Foundation Grant TBF1473. 

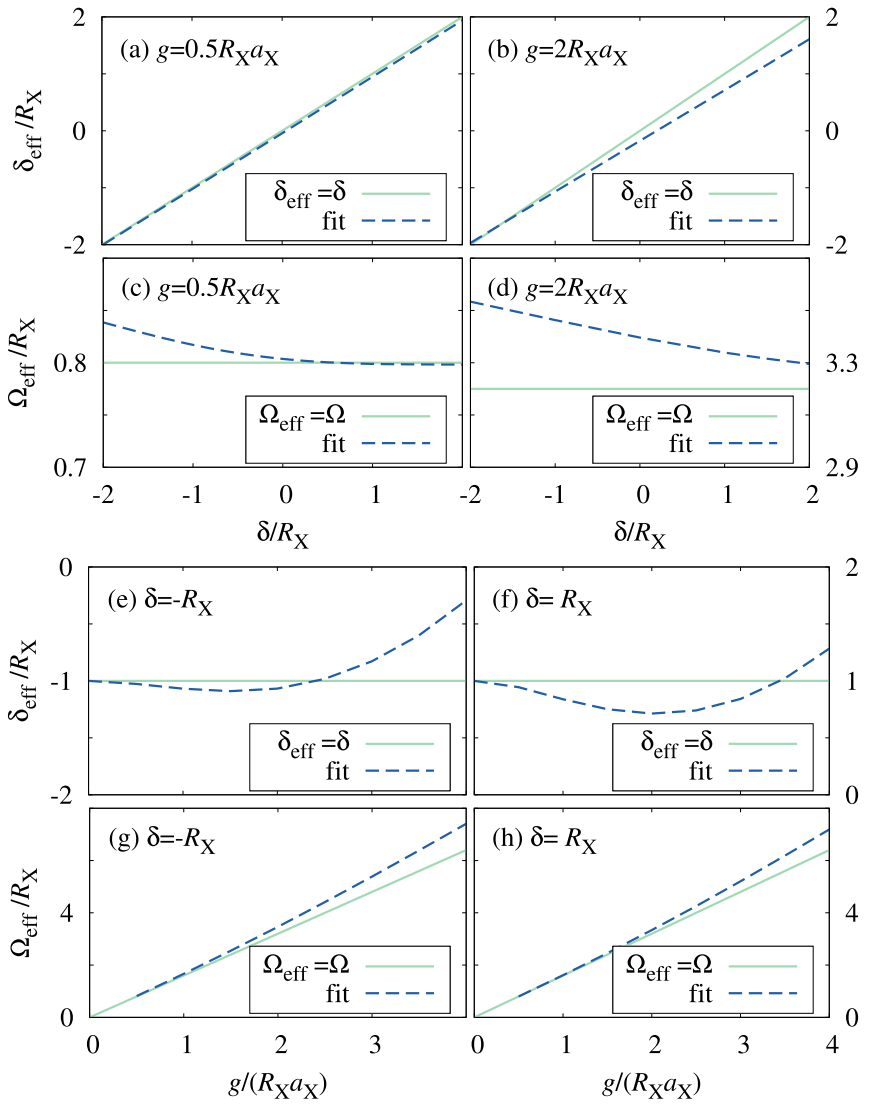

FIG. 9. Comparison between detuning $\delta$ (12) and Rabi splitting $\Omega$ (13), as defined in the renormalization procedure of Sec. II A, and the respective quantities $\delta_{\text {eff }}$ and $\Omega_{\text {eff }}$ obtained by a least-squares fitting procedure described in Appendix A. Parameters are for a GaAs microcavity with a single quantum well $(d=0), m_{2} / m_{1}=$ 0.25 , and $E_{\mathrm{F}}=0$.

This work was performed in part at Aspen Center for Physics, which is supported by National Science Foundation Grant No. PHY-1607611. This work was partially supported by a grant from the Simons Foundation.

\section{APPENDIX A: VALIDITY OF THE RENORMALIZATION PROCEDURE BEYOND THE $g \ll a_{\mathrm{X}} R_{\mathrm{X}}$ LIMIT}

We discuss here an improvement of the renormalization procedure employed in the main text to increase its accuracy beyond the weak-coupling limit. In Sec. II A we saw that in the weak-coupling limit $g \ll a_{\mathrm{X}} R_{\mathrm{X}}$, defining the renormalized photon-exciton detuning $\delta$ as in Eq. (12) and the Rabi splitting $\Omega$ as in Eq. (13) enables one to recover the one-particle LP energy of the coupled oscillator model, Eq. (14). Beyond weak coupling, the exciton wave function is strongly modified by light-matter coupling, thus impacting the detuning and the Rabi splitting. Here, we provide alternative definitions for the effective detuning $\delta_{\text {eff }}$ and Rabi splitting $\Omega_{\text {eff }}$ that coincide with the previous ones for $g \ll a_{\mathrm{X}} R_{\mathrm{X}}$, but whose validity extends beyond this limit. Comparing the two results allows one to estimate the quantitative error made in our study of the evolution of the system phase diagram with increasing Rabi splitting $\Omega$; see Sec. IV A.
To renormalize the theory, it is necessary to identify a measurable quantity that can be used to define the renormalized quantities in the theory. Ideally, the quantity we would use would be the photon energy. However, this is not directly measurable, since the renormalization only occurs for a cavity that contains an active medium, and in that case the photon mode is replaced by the strongly coupled polariton modes. To circumvent this problem, as in Ref. [43], we define the effective detuning $\delta_{\text {eff }}$ and Rabi splitting $\Omega_{\text {eff }}$ in a way analogous to an experimental procedure-by fitting the polariton dispersion to a coupled oscillator model.

In particular, we employ a two-parameter least-squares fitting procedure to match the LP dispersion $E_{\mathbf{Q}}$ evaluated numerically from Eqs. (17) with the LP dispersion obtained by the coupled oscillator model (14),

$$
\begin{aligned}
\omega_{\mathrm{LPQ}}= & E_{\mathrm{X}}^{(d)}+E_{g}+\frac{\delta_{\mathrm{eff}}+\frac{\mathbf{Q}^{2}}{2 m_{\mathrm{C}}}+\frac{\mathbf{Q}^{2}}{2\left(m_{1}+m_{2}\right)}}{2} \\
& -\frac{1}{2} \sqrt{\left(\delta_{\mathrm{eff}}+\frac{\mathbf{Q}^{2}}{2 m_{\mathrm{C}}}-\frac{\mathbf{Q}^{2}}{2\left(m_{1}+m_{2}\right)}\right)^{2}+\Omega_{\mathrm{eff}}^{2}},
\end{aligned}
$$

where $\delta_{\text {eff }}$ and $\Omega_{\text {eff }}$ are fitting parameters. In Fig. 9 we compare the results obtained for the fitting parameters $\delta_{\text {eff }}$ and $\Omega_{\text {eff }}$ with $\delta$ and $\Omega$ as defined in Eqs. (12) and (13), respectively. In Figs. 9(a)-9(d) we fix the light-matter coupling $g$ and vary $\delta$, while in Figs. 9(e)-9(h) we fix $\delta$ and vary $g$. As expected, $\delta_{\text {eff }} \rightarrow \delta$ and $\Omega_{\text {eff }} \rightarrow \Omega$ when $g \ll R_{\mathrm{X}} a_{\mathrm{X}}$. Moreover, we observe that the differences $\left|\delta_{\text {eff }}-\delta\right|$ and $\Omega_{\text {eff }}-\Omega$ remain relatively small also when $g \gtrsim R_{\mathrm{X}} a_{\mathrm{X}}$. These results allow us to estimate the size of the corrections that would arise from an improved renormalization scheme. We see that these appear small. Nonetheless, there may be some changes in the results of Sec. IV A when studying the phase diagram beyond the $g \ll R_{\mathrm{X}} a_{\mathrm{X}}$ regime.

\section{APPENDIX B: EXCITON ENERGIES AT FINITE $\boldsymbol{E}_{\mathrm{F}}$}

In Fig. 10, we compare the density dependence behavior of the rescaled energies $E_{\mathrm{XQ}}^{\left(d, E_{\mathrm{F}}\right)}-E_{\mathrm{X}}^{(d)}$ of the many-body exciton state at $\mathbf{Q}=\mathbf{0}$ (solid line) and at $\mathbf{Q}_{\min }$ (dashed line) for different mass ratios $m_{2} / m_{1}=0.25,4$ and for both screened and unscreened interactions. Note that while the dependence of $E_{\mathrm{X} \boldsymbol{0}}^{\left(d, E_{\mathrm{F}}\right)}$ on $E_{\mathrm{F}}$ is sublinear for small values of $E_{\mathrm{F}}$ and screened interactions, it eventually becomes linear at large $E_{\mathrm{F}}$.

In Fig. 11 we plot $E_{\mathrm{XQ}}^{\left(d, E_{\mathrm{F}}\right)}-E_{\mathrm{X}}^{(d)}$ as a function of density for a specific choice of parameters and superimpose a color map of the photon fraction $\left|\alpha_{0}\right|^{2}$ of the many-body $\mathbf{Q}=\mathbf{0}$ polariton state as a function of $E_{F}$ and detuning $\delta$. The red region shows where the photon fraction is around 50\% indicating that the cavity photon energy is resonant with the many-body $\mathbf{Q}=\mathbf{0}$ exciton state-see Eqs. (24) and (25). As discussed is Sec. IIIC, the photon energy shift at $\mathbf{Q}=\mathbf{0}$, $\omega_{\mathrm{C} 0}^{\left(E_{\mathrm{F}}\right)}-\omega_{\mathrm{C} \boldsymbol{0}}$, depends only weakly on $E_{\mathrm{F}}$. In particular, for the small value of $\Omega$ used in Fig. $11\left(\Omega=0.2 R_{\mathrm{X}}\right)$, we expect that the $E_{\mathrm{F}}$ dependence of the effective photon energy is negligible with respect to that of the exciton energy, $\mid \omega_{\mathrm{C} 0}^{\left(E_{\mathrm{F}}\right)}-$ $\omega_{\mathrm{C} \mathbf{0}}|\ll| E_{\mathrm{X} \boldsymbol{F}}^{\left(d, E_{\mathrm{F}}\right)}-E_{\mathrm{X}}^{(d)} \mid$. Thus, in this case, we expect that $\delta_{50 \%} \simeq E_{\mathrm{X} \mathbf{0}}^{\left(d, E_{\mathrm{F}}\right)}-E_{\mathrm{X}}^{(d)}$, which matches what is observed in Fig. 11: The detuning $\delta$ at which resonance occurs (red region) 


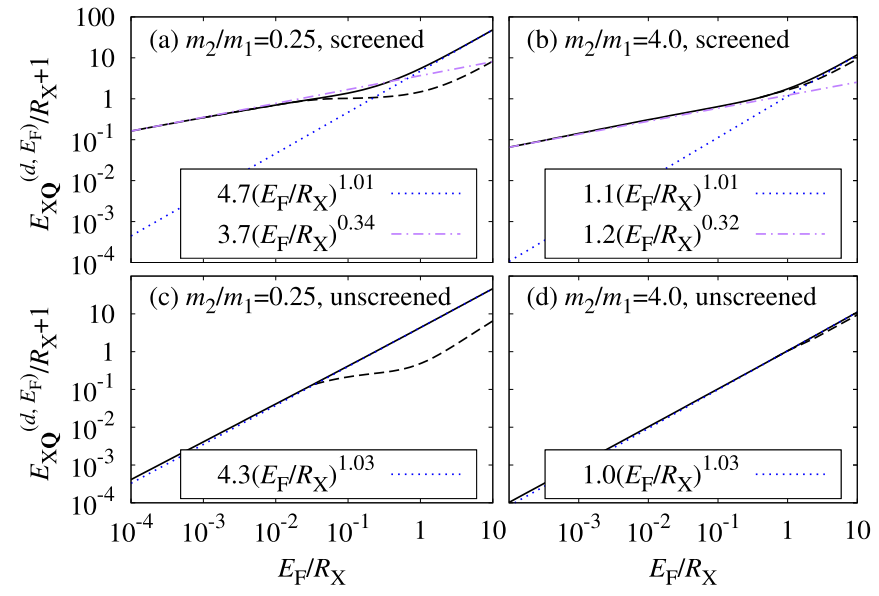

FIG. 10. Rescaled shift of exciton energies $E_{\mathrm{XQ}}^{\left(d, E_{\mathrm{F}}\right)}-E_{\mathrm{X}}^{(d)}$ at $\mathbf{Q}=$ $\mathbf{0}$ (solid) and $\mathbf{Q}_{\min }$ (dashed) as a function of density. Parameters are for a GaAs single quantum well $\left(d=0, E_{\mathrm{X}}^{(d=0)}=-R_{\mathrm{X}}\right)$, two different mass ratios $m_{2} / m_{1}=0.25$ and 4 , and for both screened $\left(N_{s}=1\right)$ [panels (a),(b)] and unscreened $\left(N_{s}=0\right)$ [panels (c),(d)] interactions. Dot-dashed and dotted lines show the low- and highdensity fittings, respectively.

coincides with the energy shift of the exciton, $E_{\mathrm{X} \mathbf{0}}^{\left(d, E_{\mathrm{F}}\right)}-E_{\mathrm{X}}^{(d)}$ (solid line).

\section{APPENDIX C: FIRST- VERSUS SECOND-ORDER TRANSITIONS}

As shown in Ref. [10], in the absence of the photon field, the excitonic SF-FF transition is always second order. In Fig. 12 we show this by plotting the momentum $Q_{\min }-$ which minimizes the many-body exciton energy $E_{\mathbf{Q}}=E_{\mathrm{XQ}}^{\left(d, E_{\mathrm{F}}\right)}$

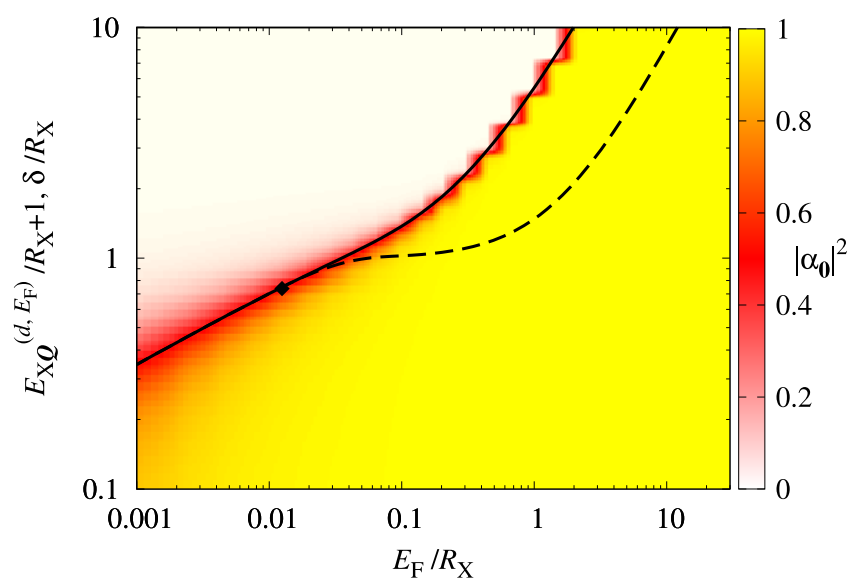

FIG. 11. Rescaled shift of exciton energies $E_{\mathrm{XQ}}^{\left(d, E_{\mathrm{F}}\right)}-E_{\mathrm{X}}^{(d)}$ at $\mathbf{Q}=$ $\mathbf{0}$ (solid) and $\mathbf{Q}_{\min }$ (dashed) as a function of the density. Parameters are for a GaAs single quantum well $\left(d=0, E_{\mathrm{X}}^{(d=0)}=-R_{\mathrm{X}}\right)$, $m_{2} / m_{1}=0.25$, and screened interactions $N_{s}=1$. The diamond symbol represents the Fermi energy $E_{\mathrm{F} 0}$ at which the two energies split as a consequence of a second-order transition where $\mathbf{Q}_{\text {min }}$ moves away from $\mathbf{0}$. The color map is the photon fraction $\left|\alpha_{0}\right|^{2}$ of the many-body polariton state at $\mathbf{Q}=\mathbf{0}$ for $\Omega=0.2 R_{\mathrm{X}}$. The color map is plotted against $E_{F} / R_{\mathrm{X}}\left(x\right.$-axis) and detuning $\delta / R_{\mathrm{X}}(y$-axis).
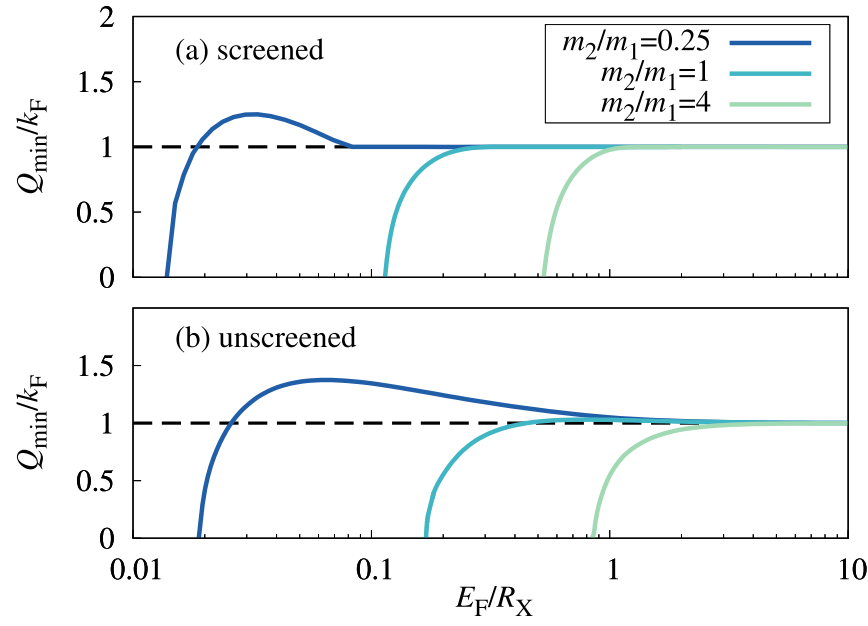

FIG. 12. Momentum $Q_{\min }$ minimizing the many-body exciton energy $E_{\mathrm{Q}}=E_{\mathrm{XQ}}^{\left(d, E_{\mathrm{F}}\right)}$ obtained by solving Eq. (18) as a function of the majority Fermi energy $E_{\mathrm{F}}$ for a single quantum well $(d=0)$, and for different values of the mass ratios $m_{2} / m_{1}$ as indicated. In panel (a) the interaction is screened, $N_{s}=1$, while in panel (b) it is unscreened, $N_{s}=0$.

solution of Eq. (18) — as a function of the Fermi energy of the majority species. We see that the transition from the $\mathrm{SF} \mathbf{Q}=\mathbf{0}$ to the finite momentum FF phase is continuous. In addition, for screened interactions, when increasing the density further, $Q_{\text {min }}$ locks to precisely $k_{\mathrm{F}}$ at the FF-N transition.

In the presence of a cavity field, the transitions SF-FF and SF-N are instead first order. This is shown in Fig. 13, where we plot the energy of the polaritonic state versus $Q$. These data refer to the parameters of Figs. 4 and 5. In panel (a) we show three curves varying the majority species density close to the first SF-FF transition. We have taken a positive large value of the detuning at $E_{\mathrm{F}}=0, \delta=8 R_{\mathrm{X}}$, such that the photon energy is far above the range of energies shown in this figure. Nevertheless, by comparing the many-body LP energy Q-dispersion with that of the many-body exciton (dashed lines, corresponding to $\Omega=0$ ), we observe significant effects of mixing between light and matter near $\mathbf{Q}=\mathbf{0}$. The lightmatter mixing at $Q_{\min }$ is much smaller, around $\left|\alpha_{Q_{\min }}\right|^{2} \simeq$ $10^{-6}$. As a result, the energy shows two local minima that cross - the signature of a first-order transition. For the NSF transition that occurs at larger majority species density, Fig. 13(b), we observe that there is minimal coupling between matter and light both for the $\mathrm{SF} \mathbf{Q}=\mathbf{0}$ state (because the $\mathbf{Q}=$ $\mathbf{0}$ exciton energy state is pushed here to very high energies by Pauli blocking) and for the normal state at $Q_{\min }=k_{\mathrm{F}}$, which has zero photon fraction.

\section{APPENDIX D: ORIGIN OF $E_{\mathrm{F}}^{*}$ AND $\delta^{*}$}

We explain here the origin of the "universal point" $\left(E_{\mathrm{F}}^{*}, \delta^{*}\right)$ found in the phase diagram of Fig. 6. Remarkably, exactly at this point there is no $\Omega$ dependence of either the SF-FF transition (for unscreened interactions) or the SF-N transition (for screened interactions). One way to understand the origin of this universal point is by comparing the many-body LP energy of the SF state at $\mathbf{Q}=\mathbf{0}, E_{\mathbf{0}}$, with that of the FF 

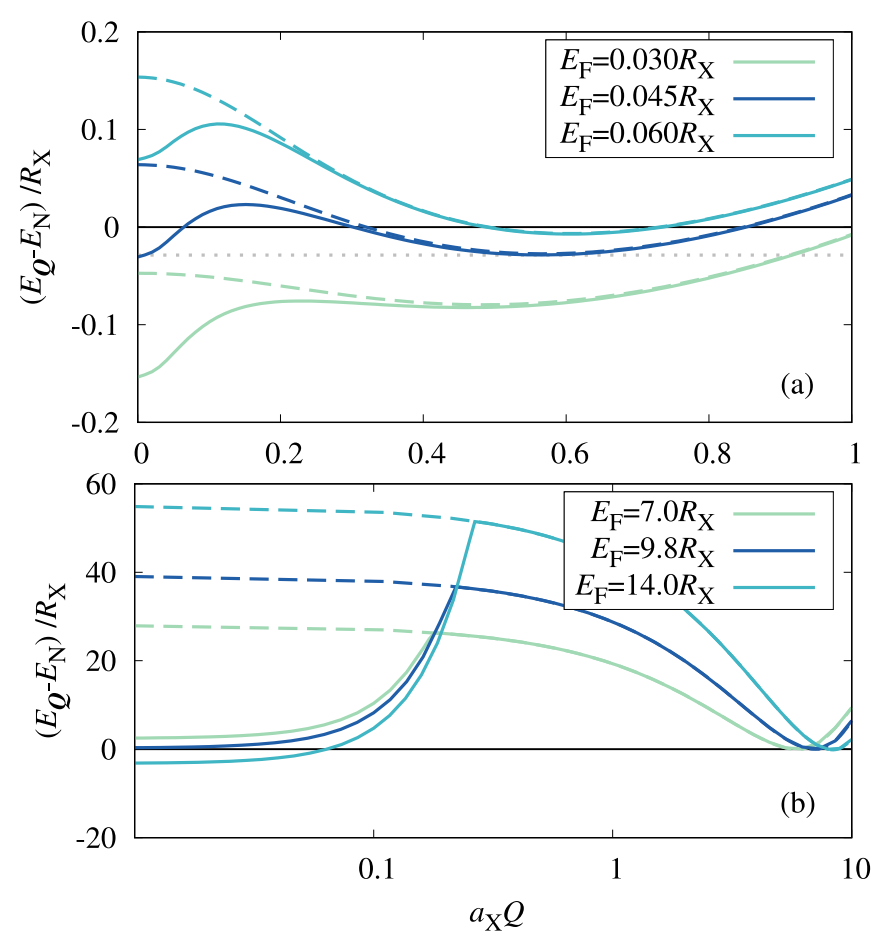

FIG. 13. Many-body polariton ground-state energy $E_{\mathbf{Q}}$ with respect to the normal-state energy $E_{\mathrm{N}}$ (solid lines) vs momentum $Q$. Parameters are for a GaAs heterostructure with a single quantum well $(d=0)$, mass ratio $m_{2} / m_{1}=0.25$, Rabi splitting $\Omega=2 R_{\mathrm{X}}$, detuning $\delta=8 R_{\mathrm{X}}$, and screened interactions $\left(N_{s}=1\right)$. Dashed colored lines are the many-body exciton energies $E_{\mathrm{XQ}}^{\left(d, E_{\mathrm{F}}\right)}$ evaluated in the absence of the light-matter coupling, $\Omega=0$. The gray dotted line indicates where the minima at $Q=0$ and $Q \neq 0$ are equal. Panel (a) shows the first-order SF-FF transition when increasing the system density, while panel (b) shows the N-SF first-order transition.

phase at $Q_{\min }, E_{Q_{\min }}$. The two energies clearly coincide at this first-order boundary (for screened interactions, the FF phase may be replaced by the $\mathrm{N}$ phase if the density is large enough). A limiting case of this boundary occurs when $\Omega \rightarrow 0$; in this limit, the boundary occurs when

$$
\delta=E_{\mathrm{X} \mathbf{Q}_{\text {min }}}^{\left(d, E_{\mathrm{F}}\right)}-E_{\mathrm{X}}^{(d)}
$$

(assuming $E_{\mathrm{F}}>E_{\mathrm{F} 0}$ ), where $E_{\mathrm{X}}^{\left(d, E_{\mathrm{F}}\right)}$ is the many-body exciton (i.e., $\Omega=0$ case) energy of the $\mathrm{FF}$ phase for a majority species Fermi energy $E_{\mathrm{F}}$. This condition corresponds to a crossing between a photonic SF state and the excitonic FF state. At nonzero $\Omega$, the SF state becomes polaritonic.

The existence of the special point $\left(E_{\mathrm{F}}^{*}, \delta^{*}\right)$ corresponds to a point where this critical condition is not affected by light-matter coupling. To see this, we consider the following. At each $E_{\mathrm{F}}$, we can choose the detuning $\delta$ so as to satisfy Eq. (D1), thus on the SF-FF boundary at $\Omega=0$. We then plot in Figs. 14(a) and 14(b) $E_{\mathbf{0}}-E_{\mathbf{Q}_{\min }}$, the energy difference between the LP energy at $\mathbf{Q}=\mathbf{0}$ and $\mathbf{Q}=\mathbf{Q}_{\min }$, as a function of $\Omega$. We plot this energy difference for different values of $E_{\mathrm{F}}$. For $E_{\mathrm{F}}<E_{\mathrm{F}}^{*}$, this energy difference decreases with $\Omega$. This means that upon increasing $\Omega$, the SF-FF boundary moves to larger values of the detuning (see Fig. 6). Conversely, if $E_{\mathrm{F}}>E_{\mathrm{F}}^{*}$, the energy difference increases with $\Omega$, so the SF-FF
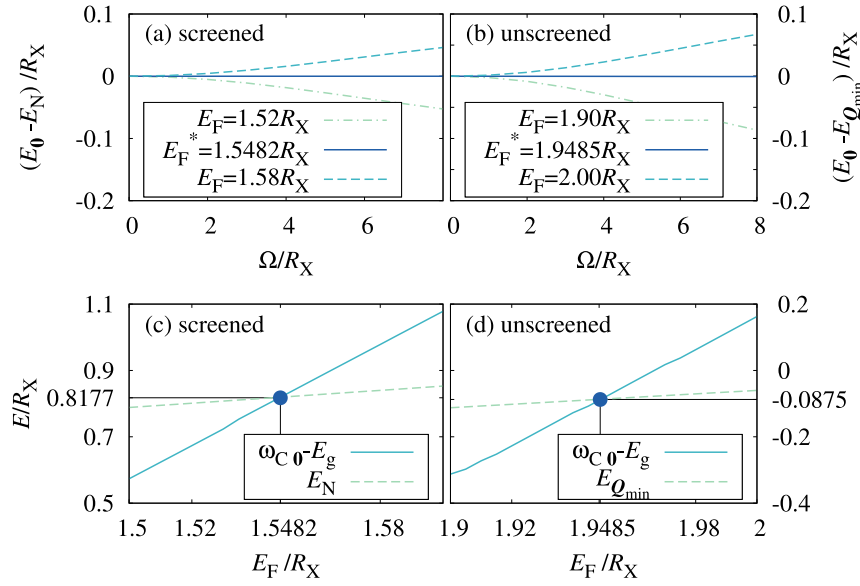

FIG. 14. Illustration of the dependence of energies on Rabi splitting close to $E_{\mathrm{F}}^{*}$. The parameters are for a GaAs heterostructures with a single quantum well $d=0$, mass ratio $m_{2} / m_{1}=0.25$, and for screened [panels (a) and (c)] and unscreened [panels (b) and (d)] interactions. (a) and (b) Energy difference between the many-body polariton SF energy $E_{\mathbf{0}}$ and the FF energy $E_{\mathbf{Q}_{\min }}$ [panel (b)] or between $E_{0}$ and the normal-state energy $E_{\mathrm{N}}$ [panel (a)]. For each Fermi energy, $E_{\mathrm{F}}$, the detuning is fixed according to Eq. (D1), describing the SF-FF boundary at $\Omega=0$. (c) and (d) Photon energy $\omega_{\mathrm{C} 0}-E_{g}$ satisfying Eq. (D9) at $\mathbf{Q}=\mathbf{0}$ and $E_{\mathbf{Q}_{\min }}$-for the values of $E_{\mathrm{F}}$ considered in the plot and for screened interactions, $E_{\mathbf{Q}_{\min }}$ coincides with the normal-state energy $E_{\mathrm{N}}$, i.e., $\mathbf{Q}_{\min }=k_{\mathrm{F}} \hat{\mathbf{k}}_{\mathrm{F}}$.

boundary moves down to lower detuning. Exactly at $E_{\mathrm{F}}=E_{\mathrm{F}}^{*}$, we observe that $E_{\mathbf{0}}-E_{\mathbf{Q}_{\min }}=0$ becomes exactly independent of $\Omega$. As such, at this value of $E_{\mathrm{F}}^{*}$, the critical detuning is $\delta^{*}$, independent of $\Omega$.

Given the effective $\Omega$ independence seen at $E_{\mathrm{F}}^{*}$, an alternative way of identifying the value of $E_{\mathrm{F}}^{*}$ and $\delta^{*}$ is by finding a condition for which the eigenenergy of the variational state becomes independent of the coupling to light. To do this, following Ref. [43], we rewrite the many-body eigenvalue problem of Eqs. (17) in terms of the renormalized photon energy $\omega_{\mathrm{CQ}}=\omega_{\mathrm{C} \mathbf{0}}+\mathbf{Q}^{2} / 2 m_{\mathrm{C}}$ (11) to give an expression that is independent of the UV cutoff. We thus separate out the divergent part of the relative wave function $\varphi_{\mathbf{k} \mathbf{Q}}$,

$$
\varphi_{\mathbf{k} \mathbf{Q}}=\beta_{\mathbf{k} \mathbf{Q}}+\frac{g \alpha_{\mathbf{Q}}}{E_{\mathbf{Q}}-\xi_{\mathbf{k} \mathbf{Q}}},
$$

and we rewrite (17) in the following equivalent forms:

$$
\begin{aligned}
& \left(E_{\mathbf{Q}}-\xi_{\mathbf{k} \mathbf{Q}}\right) \beta_{\mathbf{k} \mathbf{Q}}=-\frac{1}{\mathcal{A}} \sum_{\mathbf{k}^{\prime}>k_{\mathrm{F}}} V_{\mathbf{k}-\mathbf{k}^{\prime}} \beta_{\mathbf{k}^{\prime} \mathbf{Q}}+\frac{g \alpha_{\mathbf{Q}}}{\mathcal{A}} \sum_{\mathbf{k}^{\prime}>k_{\mathrm{F}}} \frac{V_{\mathbf{k}-\mathbf{k}^{\prime}}}{-E_{\mathbf{Q}}+\xi_{\mathbf{k}^{\prime} \mathbf{Q}}} \\
& {\left[E_{\mathbf{Q}}-\omega_{\mathrm{CQ}}+E_{g}+\frac{g^{2}}{\mathcal{A}}\left(\sum_{\mathbf{k}>k_{\mathrm{F}}} \frac{1}{-E_{\mathbf{Q}}+\xi_{\mathbf{k} \mathbf{Q}}}\right.\right.} \\
& \left.\left.-\sum_{\mathbf{k}} \frac{1}{-E_{\mathrm{X}}^{(d)}+\epsilon_{\mathbf{k}, 1}+\epsilon_{\mathbf{k}, 2}}\right)\right] \alpha_{\mathbf{Q}}=\frac{g}{\mathcal{A}} \sum_{\mathbf{k}>k_{\mathrm{F}}} \beta_{\mathbf{k} \mathbf{Q}}
\end{aligned}
$$

All sums are now convergent. For the solution of these equations to be independent of light-matter coupling means the $E_{\mathbf{Q}}$ 
must match the solution at $g=0$, i.e.,

$$
E_{\mathbf{Q}}=\omega_{\mathrm{CQ}}-E_{g} .
$$

This condition corresponds to the system energy $E_{\mathbf{Q}}+E_{g}$ coinciding with $\omega_{\mathrm{CQ}}$, the energy of the photon mode at $E_{\mathrm{F}}=$ 0. Using Eq. (D4) in Eq. (D3b), we obtain the following equation to define $E_{\mathrm{F}}^{*}$ :

$\sum_{\mathbf{k}>k_{\mathrm{F}}} \frac{1}{-E_{\mathbf{Q}}+\xi_{\mathbf{k} \mathbf{Q}}}-\sum_{\mathbf{k}} \frac{1}{-E_{\mathrm{X}}^{(d)}+\epsilon_{\mathbf{k}, 1}+\epsilon_{\mathbf{k}, 2}}=\frac{1}{g \alpha_{\mathbf{Q}}} \sum_{\mathbf{k}>k_{\mathrm{F}}} \beta_{\mathbf{k} \mathbf{Q}}$.

Note that this condition is indeed independent of $g$. To see this, we formally invert Eq. (D3a) to give $\beta_{\mathbf{k} \mathbf{Q}}$ :

$$
\beta_{\mathbf{k} \mathbf{Q}}=g \alpha_{\mathbf{Q}} \sum_{\mathbf{k}^{\prime}>k_{\mathrm{F}}}\left(\mathbb{M}_{\mathbf{Q}}{ }^{-1}\right)_{\mathbf{k}, \mathbf{k}^{\prime}} L_{\mathbf{k}^{\prime} \mathbf{Q}}
$$

where the matrix $\mathbb{M}_{\mathbf{Q}}$ and vector $L_{\mathbf{Q}}$ in relative momentum space are defined, respectively, as

$$
\begin{aligned}
\left(\mathbb{M}_{\mathbf{Q}}\right)_{\mathbf{k}, \mathbf{k}^{\prime}} & =\left(E_{\mathbf{Q}}-\xi_{\mathbf{k} \mathbf{Q}}\right) \delta_{\mathbf{k}, \mathbf{k}^{\prime}}+V_{\mathbf{k}-\mathbf{k}^{\prime}}, \\
L_{\mathbf{k} \mathbf{Q}} & =\sum_{\mathbf{k}^{\prime}>k_{\mathrm{F}}} \frac{V_{\mathbf{k}-\mathbf{k}^{\prime}}}{-E_{\mathbf{Q}}+\xi_{\mathbf{k}^{\prime} \mathbf{Q}}} .
\end{aligned}
$$

[1] T. Brunhes, R. André, A. Arnoult, J. Cibert, and A. Wasiela, Phys. Rev. B 60, 11568 (1999).

[2] R. Rapaport, E. Cohen, A. Ron, E. Linder, and L. N. Pfeiffer, Phys. Rev. B 63, 235310 (2001).

[3] A. Qarry, R. Rapaport, G. Ramon, E. Cohen, A. Ron, and L. N. Pfeiffer, Semicond. Sci. Technol. 18, S331 (2003).

[4] D. Bajoni, M. Perrin, P. Senellart, A. Lemaître, B. Sermage, and J. Bloch, Phys. Rev. B 73, 205344 (2006).

[5] A. Gabbay, Y. Preezant, E. Cohen, B. M. Ashkinadze, and L. N. Pfeiffer, Phys. Rev. Lett. 99, 157402 (2007).

[6] S. Smolka, W. Wuester, F. Haupt, S. Faelt, W. Wegscheider, and A. Imamoglu, Science 346, 332 (2014).

[7] M. Sidler, P. Back, O. Cotlet, A. Srivastava, T. Fink, M. Kroner, E. Demler, and A. Imamoglu, Nat. Phys. 13, 255 (2016).

[8] R. P. A. Emmanuele, M. Sich, O. Kyriienko, V. Shahnazaryan, F. Withers, A. Catanzaro, P. M. Walker, F. A. Benimetskiy, M. S. Skolnick, A. I. Tartakovskii, I. A. Shelykh, and D. N. Krizhanovskii, arXiv:1910.14636.

[9] A. L. Subası, P. Pieri, G. Senatore, and B. Tanatar, Phys. Rev. B 81, 075436 (2010).

[10] M. M. Parish, F. M. Marchetti, and P. B. Littlewood, Europhys. Lett. 95, 27007 (2011).

[11] J. R. Varley and D. K. K. Lee, Phys. Rev. B 94, 174519 (2016).

[12] O. Cotlet, D. S. Wild, M. D. Lukin, and A. Imamoglu, arXiv:1812.10494.
We thus find that Eq. (D5) is independent of both $g$ and $\alpha_{\mathbf{Q}}$ :

$$
\begin{aligned}
& \sum_{\mathbf{k}>k_{\mathrm{F}}} \frac{1}{-E_{\mathbf{Q}}+\xi_{\mathbf{k} \mathbf{Q}}}-\sum_{\mathbf{k}} \frac{1}{-E_{\mathrm{X}}^{(d)}+\epsilon_{\mathbf{k}, 1}+\epsilon_{\mathbf{k}, 2}} \\
& =\sum_{\mathbf{k}>k_{\mathrm{F}}} \sum_{\mathbf{k}^{\prime}>k_{\mathrm{F}}}\left(\mathbb{M}_{\mathbf{Q}}{ }^{-1}\right)_{\mathbf{k}, \mathbf{k}^{\prime}} L_{\mathbf{k}^{\prime} \mathbf{Q}} .
\end{aligned}
$$

In addition to satisfying Eq. (D9), $E_{\mathrm{F}}^{*}$ lies on the SF-FF (SF-N) boundaries for unscreened (screened) interactions, and thus it also has to lie on the boundary at $\Omega=0$. With this in mind, we plot in Figs. 14(c) and 14(d) the energy $\omega_{\mathrm{C} 0}-E_{g}=$ $E_{\mathbf{0}}$ obtained by solving Eq. (D9) at $\mathbf{Q}=\mathbf{0}$ as a function of $E_{\mathrm{F}}$. From the crossing of this curve with that of the FF state in the absence of light, i.e., the FF exciton energy $E_{\mathbf{X} \mathbf{Q}_{\min }}^{\left(d, E_{\mathrm{F}}\right)}$ (or, for the screened case, the normal state energy $E_{\mathrm{N}}$ ), we recover the value of $E_{\mathrm{F}}^{*}$. The corresponding value of the detuning $\delta^{*}$ is given by Eq. (D1) for $E_{\mathrm{F}}=E_{\mathrm{F}}^{*}$, i.e., $\delta^{*}=E_{\mathrm{X}}^{\left(d, E_{\mathrm{Fin}}^{*}\right)}-$ $E_{\mathrm{X}}^{(d)}$. We thus find $\left(E_{\mathrm{F}}^{*}, \delta^{*}\right) \simeq\left(1.55 R_{\mathrm{X}}, 1.82 R_{\mathrm{X}}\right)$ (for screened interactions) and $\left(E_{\mathrm{F}}^{*}, \delta^{*}\right) \simeq\left(1.95 R_{\mathrm{X}}, 0.91 R_{\mathrm{X}}\right)$ (unscreened interactions).

Finally, we remark that the $g$ independence at $E_{\mathrm{F}}^{*}$ does not imply that light and matter are fully decoupled at this point. Indeed, the photon frequency depends on the active medium through the process of renormalization. However, precisely at $E_{\mathrm{F}}^{*}$, the photon self-energy arising due to the light-matter interaction only contains the term that appears in Eq. (11), while all other terms cancel. Given the general arguments that led us to determine the point $\left(E_{\mathrm{F}}^{*}, \delta^{*}\right)$, it is likely that it persists as a special point in the photon self energy also beyond the variational approach used in this work.
[13] P. Fulde and R. A. Ferrell, Phys. Rev. 135, A550 (1964),

[14] A. I. Larkin and Y. N. Ovchinnikov, Zh. Eksp. Teor. Fiz. 47, 1136 (1964) [Sov. Phys. JETP 20, 762 (1965)].

[15] R. Casalbuoni and G. Nardulli, Rev. Mod. Phys. 76, 263 (2004).

[16] L. Radzihovsky and D. E. Sheehy, Rep. Prog. Phys. 73, 076501 (2010).

[17] J. J. Kinnunen, J. E. Baarsma, J.-P. Martikainen, and P. Törmä, Rep. Prog. Phys. 81, 046401 (2018).

[18] A. Bianchi, R. Movshovich, C. Capan, P. G. Pagliuso, and J. L. Sarrao, Phys. Rev. Lett. 91, 187004 (2003).

[19] S. Uji, T. Terashima, M. Nishimura, Y. Takahide, T. Konoike, K. Enomoto, H. Cui, H. Kobayashi, A. Kobayashi, H. Tanaka, M. Tokumoto, E. S. Choi, T. Tokumoto, D. Graf, and J. S. Brooks, Phys. Rev. Lett. 97, 157001 (2006).

[20] R. Lortz, Y. Wang, A. Demuer, P. H. M. Böttger, B. Bergk, G. Zwicknagl, Y. Nakazawa, and J. Wosnitza, Phys. Rev. Lett. 99, 187002 (2007).

[21] R. Beyer, B. Bergk, S. Yasin, J. A. Schlueter, and J. Wosnitza, Phys. Rev. Lett. 109, 027003 (2012).

[22] G. Koutroulakis, H. Kühne, J. A. Schlueter, J. Wosnitza, and S. E. Brown, Phys. Rev. Lett. 116, 067003 (2016).

[23] S. Sugiura, T. Isono, T. Terashima, S. Yasuzuka, J. A. Schlueter, and S. Uji, npj Quantum Mater. 4, 7 (2019).

[24] C.-W. Cho, J. H. Yang, N. F. Q. Yuan, J. Shen, T. Wolf, and R. Lortz, Phys. Rev. Lett. 119, 217002 (2017). 
[25] Y.-a. Liao, A. S. C. Rittner, T. Paprotta, W. Li, G. B. Partridge, R. G. Hulet, S. K. Baur, and E. J. Mueller, Nature (London) 467, 567 (2010).

[26] K. F. Mak and J. Shan, Nat. Photon. 10, 216 (2016).

[27] T. C. Berkelbach, M. S. Hybertsen, and D. R. Reichman, Phys. Rev. B 88, 045318 (2013).

[28] P. Cudazzo, L. Sponza, C. Giorgetti, L. Reining, F. Sottile, and M. Gatti, Phys. Rev. Lett. 116, 066803 (2016).

[29] A. Chernikov, A. M. van der Zande, H. M. Hill, A. F. Rigosi, A. Velauthapillai, J. Hone, and T. F. Heinz, Phys. Rev. Lett. 115, 126802 (2015).

[30] Z. Wang, D. A. Rhodes, K. Watanabe, T. Taniguchi, J. C. Hone, J. Shan, and K. F. Mak, Nature (London) 574, 76 (2019).

[31] X. Liu, T. Galfsky, Z. Sun, F. Xia, E.-c. Lin, Y.-H. Lee, S. KénaCohen, and V. M. Menon, Nat. Photon. 9, 30 (2015).

[32] Y.-J. Chen, J. D. Cain, T. K. Stanev, V. P. Dravid, and N. P. Stern, Nat. Photon. 11, 431 (2017).

[33] Z. Sun, J. Gu, A. Ghazaryan, Z. Shotan, C. R. Considine, M. Dollar, B. Chakraborty, X. Liu, P. Ghaemi, S. Kéna-Cohen, and V. M. Menon, Nat. Photon. 11, 491 (2017).

[34] X. Liu, W. Bao, Q. Li, C. Ropp, Y. Wang, and X. Zhang, Phys. Rev. Lett. 119, 027403 (2017).

[35] U. Sivan, P. M. Solomon, and H. Shtrikman, Phys. Rev. Lett. 68, 1196 (1992).

[36] A. F. Croxall, K. Das Gupta, C. A. Nicoll, M. Thangaraj, H. E. Beere, I. Farrer, D. A. Ritchie, and M. Pepper, Phys. Rev. Lett. 101, 246801 (2008).

[37] J. A. Seamons, C. P. Morath, J. L. Reno, and M. P. Lilly, Phys. Rev. Lett. 102, 026804 (2009).

[38] G. Mahan, Many-Particle Physics, Physics of Solids and Liquids (Springer, New York, 2013).

[39] D. Pimenov, J. von Delft, L. Glazman, and M. Goldstein, Phys. Rev. B 96, 155310 (2017).

[40] D. K. Efimkin and A. H. MacDonald, Phys. Rev. B 95, 035417 (2017).

[41] X. L. Yang, S. H. Guo, F. T. Chan, K. W. Wong, and W. Y. Ching, Phys. Rev. A 43, 1186 (1991).

[42] H. Deng, H. Haug, and Y. Yamamoto, Rev. Mod. Phys. 82, 1489 (2010).

[43] J. Levinsen, G. Li, and M. M. Parish, Phys. Rev. Research 1, 033120 (2019).

[44] S. Brodbeck, S. De Liberato, M. Amthor, M. Klaas, M. Kamp, L. Worschech, C. Schneider, and S. Höfling, Phys. Rev. Lett. 119, 027401 (2017).
[45] A. İmamoğlu (private communication).

[46] C. Weisbuch, M. Nishioka, A. Ishikawa, and Y. Arakawa, Phys. Rev. Lett. 69, 3314 (1992).

[47] J. Bloch, T. Freixanet, J. Y. Marzin, V. Thierry-Mieg, and R. Planel, Appl. Phys. Lett. 73, 1694 (1998).

[48] M. Saba, C. Ciuti, J. Bloch, V. Thierry-Mieg, R. André, L. S. Dang, S. Kundermann, A. Mura, G. Bongiovanni, J. L. Staehli, and B. Deveaud, Nature (London) 414, 731 (2001).

[49] J. Kasprzak, M. Richard, S. Kundermann, A. Baas, P. Jeambrun, J. M. J. Keeling, F. M. Marchetti, M. H. Szymańska, R. André, J. L. Staehli, V. Savona, P. B. Littlewood, B. Deveaud, and L. S. Dang, Nature (London) 443, 409 (2006).

[50] P. Back, M. Sidler, O. Cotlet, A. Srivastava, N. Takemura, M. Kroner, and A. Imamoğlu, Phys. Rev. Lett. 118, 237404 (2017).

[51] L. V. Keldysh, Pis'ma Zh. Eksp. Teor. Fiz. 29, 716 (1979) [Sov. J. Exp. Theor. Phys. Lett. 29, 658 (1979)].

[52] N. S. Rytova, Moscow Univ. Phys. Bull. 3, 18 (1967).

[53] D. Van Tuan, M. Yang, and H. Dery, Phys. Rev. B 98, 125308 (2018).

[54] F. A. Rasmussen and K. S. Thygesen, J. Phys. Chem. C 119 13169 (2015)

[55] M. M. Ugeda, A. J. Bradley, S.-F. Shi, F. H. da Jornada, Y. Zhang, D. Y. Qiu, W. Ruan, S.-K. Mo, Z. Hussain, Z.-X. Shen, F. Wang, S. G. Louie, and M. F. Crommie, Nat. Mater. 13, 1091 (2014).

[56] S. Dufferwiel, S. Schwarz, F. Withers, A. Trichet, F. Li, M. Sich, O. Del Pozo-Zamudio, C. Clark, A. Nalitov, D. Solnyshkov et al., Nat. Commun. 6, 8579 (2015).

[57] S. Dufferwiel, T. Lyons, D. Solnyshkov, A. Trichet, F. Withers, S. Schwarz, G. Malpuech, J. Smith, K. Novoselov, M. Skolnick et al., Nat. Photon. 11, 497 (2017).

[58] J. Khurgin, Solid State Commun. 117, 307 (2001).

[59] C. Schneider, M. M. Glazov, T. Korn, S. Höfling, and B. Urbaszek, Nat. Commun. 9, 2695 (2018).

[60] M. Waldherr, N. Lundt, M. Klaas, S. Betzold, M. Wurdack, V. Baumann, E. Estrecho, A. Nalitov, E. Cherotchenko, H. Cai et al., Nat. Commun. 9, 3286 (2018).

[61] A. Strashko, F. M. Marchetti, A. H. MacDonald, and J. Keeling, arXiv:2001.07726.

[62] A. Tiene, J. Levinsen, M. M. Parish, A. H. MacDonald, J. M. J. Keeling, and F. M. Marchetti, Extremely imbalanced twodimensional electron-hole-photon systems (dataset), University of St Andrews, 2020, https://doi.org/10.17630/fc1ed8dc-298c4b4a-92da-d1e1791922a5. 